\title{
A note on the spinor construction of Spin Foam amplitudes
}

\author{
Giorgio Immirzi \\ Colle Ballone, Montopoli di Sabina (Italy) \\ e-mail: giorgio.immirzi@pg.infn.it
}

\begin{abstract}
I discuss the use of spinors in the construction of spin-foam models, in particular the form of the closure and simplicity constraints for triangles that are space-like, i.e. with (area) ${ }^{2}=\frac{1}{2} S^{I J} S_{I J}>0$, regardless of whether they belong to tetrahedra with a space-like or time-like normal, emphasizing the role of the light-like 4 -vector $u_{t} \sigma^{I} \bar{u}_{t}$. In the quantization of the model, with the representations of $\operatorname{SL}(2, \mathbb{C})$ acting on spaces of functions of light-like vectors, one may use the canonical basis of $\mathrm{SU}(2)$ representations, or the pseudobasis limited to the discrete representations of $\mathrm{SU}(1,1)$; in alternative it is proposed to use instead a basis of eigenstates of $\left(L_{3}, K_{3}\right)$, which might give matrix elements and vertex functions with the same classical limit. A detailed example of a small triangulation is presented, which among other things indicates, on the basis of a classical calculation, that it would be impractical to limit oneself to tetrahedra with time-like normals.
\end{abstract}

\section{Introduction}

In the present formulation of spin-foam theory, as it has evolved from the original BarrettCrane model [1, one associates a discretized version of the Holst action [2] $S_{\text {Holst }}=$ $\int J^{I J} \wedge F_{I J}$ with a subdivision of space-time in 4-simplices; the curvature of the connection $F_{I J}$ is replaced by the product of the $\mathrm{SL}(2, \mathbb{C})$ holonomies along each 'face' dual to a triangle (a 'bone' in Regge's language). For each triangle $t$ of a tetrahedron $\tau$ of the subdivision, $J_{t}^{I J}$ is a linear combination of the area tensor $S_{t}^{I J}$ of the triangle and of its Hodge dual ${ }^{*} S_{t}^{I J}=\frac{1}{2} \epsilon^{I J K L} S_{t K L}$ that depends on a real parameter $\gamma$ or an angle $\theta[3]$ :

$$
J_{t}^{I J}:={ }^{*} S_{t}^{I J}-\frac{1}{\gamma} S_{t}^{I J} ; \quad \gamma=-i \frac{e^{i \theta}-1}{e^{i \theta}+1} \leftrightarrow e^{i \theta}=\frac{1+i \gamma}{1-i \gamma}
$$

The area tensors $S_{t}^{I J}$ are constrained to be 'simple', the antisymmetrized product of two 4 -vectors, and to satisfy 'closure', i.e. to sum to zero for each tetrahedron.

In the quantum theory the holonomies are in a representation of $\operatorname{SL}(2, \mathbb{C})$ and the $J_{t}^{I J}$ are identified with the generators of the corresponding Lie Algebra. In the spirit of Feynman's path integral, integrating over the holonomies and summing/integrating over the representations should give a transition amplitude between an initial and a final configuration of space. The discretization breaks e.g. invariance under diffeomorphisms, but one may be optimistic, think of summing/refinining triangulations, look at the successes of lattice QCD, etc..

A lot of work and a vast literature has been dedicated to the technical problems of imposing the constraints in the quantum theory. Part of the simplicity constraints are to 
be regarded as 'second class', and the key idea of EPRL[4] is that they should be imposed on states à la Gupta-Bleuler' [5] 6].

There are however difficulties, and few detailed calculations of a transition amplitude have so far been attempted[7], while other discretized approaches, like Causal Dynamic Triangulation [8] claim great success with large simulations. One difficulty that I see is that, with the exception of [9], all the work carried on so far deals with triangulations in which all tetrahedra are assumed to be space-like, and an $\mathrm{SU}(2)$ basis is used for $\mathrm{SL}(2, \mathbb{C})$ representations. This may be too restrictive; it makes sense to assume that all triangles are space-like, without supposing that they all belong to space-like tetrahedra (i.e. with a time-like normal); this does not happen classically in a simple example I give in $\S 4$. One can follow [9] [10] and consider time-like tetrahedra as well, but at the cost of using an $\mathrm{SU}(1,1)$ basis and facing considerable tecnical difficulties. Besides, the idea that given a triangulation one should decide a priori which tetrahedra are time-like and which space-like is unpleasant. One would want an approach in which the distinction is simply irrelevant 1 .

In this note, I spell out the model in terms of spinor variables. Following [3, a pair of spinors $\left(u_{t \alpha}, t_{t \alpha}\right)$ for each triangle determine $J_{t}^{I J}$. This gives a very neat way of expressing the constraints, with no distinction between time-like or space-like tetrahedra. However, in the details of the construction, the difference does persist, although I sketch an approach to some extent unified. In fact, it turns out that what makes a difference is whether a triangle is space- or time-like, i.e. whether its (area $)^{2}$ is positive or negative, and not to what type of tetrahedron it belongs. Only positive (area) ${ }^{2}$ triangles can be described by spinor variables. In the quantum theory this implies that the labels of the representations of $\operatorname{SL}(2, \mathbb{C})$ contributing must be such that $\rho=\gamma n$, and that for triangles belonging to time-like tetrahedra one only need consider representations of $\mathrm{SU}(1,1)$ belonging to to the discrete series, with labels $k=\gamma \frac{n}{2}$. Given the normal $V_{\tau}^{I}$ to the tetrahedron, the simplicity constraints imply for each triangle a relation between $u_{t}$ and $t_{t}$, very much like the 'twistor equation' of Penrose. Using this relation one finds that the light-like 4-vector $P_{t}^{I}=u_{t} \sigma^{I} \bar{u}_{t}$ is orthogonal to the area tensor $S_{t}^{I J}$ of the triangle, and that for each tetrahedron $V_{\tau}^{I}$ is proportional to the sum of the $P_{t}^{I}$ of its four triangles, taken with signs $\kappa_{t}= \pm 1$. These signs must be such that all $\kappa_{t} P_{t}^{I}$ are either incoming or outgoing, to satisfy the closure constraint.

This representation of the geometry in terms of light-like 4-vectors coincides with the picture conjectured by Yasha Neiman[13, that he correctly supposes to be derivable from a spinor construction. And in the quantum theory I choose to realize the Hilbert spaces $\mathcal{H}^{(n, \rho)}$ on which the representations of $\mathrm{SL}(2, \mathbb{C})$ act as spaces of functions of light-like 4 -vectors ([14], pg.352).

For triangles belonging to space-like tetrahedra, hence with time-like $V_{\tau}^{I}$, one would choose the 'canonical basis' of $\mathcal{H}^{(n, \rho)}$, obtained reducing representations of $\operatorname{SL}(2, \mathbb{C})$ with respect to its $\mathrm{SU}(2)$ subgroup, made of eigenstates of $\left(\mathbf{L}^{2}, L_{3}\right)$; or for tetrahedra with spacelike $V_{\tau}^{I}$, the 'pseudobasis' obtained reducing with respect to $\mathrm{SU}(1,1)$, made of eigenstates of the $\mathrm{SU}(1,1)$ Casimir $Q=L_{3}^{2}-K_{1}^{2}-K_{2}^{2}, L_{3}$, but as I said considering only representations of the discrete series. In both cases the geometry, specifically the $P_{t}^{I}$, is encoded using coherent states of the Perelomov type; these states are obtained applying appropriate

${ }^{1}$ In Causal Dynamic Triangulation all triangles are assumed to be timelike; a possible criticism is that the Courant criterium [1] might then be systematically violated. This assumption is not modified in a recent paper 12] which relaxes the hypotheses on the foliation. 
rotations or boosts to the 'highest weight' states; imposing the constraints restricts the sums over the towers of representations to the lowest elements, $j=\frac{n}{2}$ or $k=\frac{n}{2}$.

In an attempt to treat the two cases in the same way I propose to use a 'spinor basis', made of eigenstates of the generators $\left(L_{3}, K_{3}\right)$, with eigenvalues $(m, \lambda)$. The simplicity constraints imply that we use states such that $m+\frac{1}{\gamma} \lambda=0$; applying to these states the same rotation or boost that we would have used to generate coherent states we obtain states with the wanted $P_{t}^{I}$ as expectation value. So their direction is right, but they do not have a definite area, as the eigenstates of $\mathbf{L}^{2}$ or of $Q$; calculating the overlaps between these states and eigenstates of $\left(L_{3}, K_{3}\right)$ I find they are peaked at $\lambda \approx \frac{\rho}{2}$, but I cannot tell whether this is enough to give the wanted behaviour in the semiclassical limit.

This touches on a point which has given rise to some debate: the asymptotic analysis of the 'vertex function' 15], the building block of the transition amplitude, gives a behaviour $\sim \cos ($ area $\cdot$ angle $)$ rather than the Regge $\sim e^{i \text { area angle }}$ one might have expected. Some time ago by D. Oriti and E. Livine [16] pointed out that this means that in this form the model has no causality built in; although causality is clearly what distinguishes 'euclidean' model, based on the group $\mathrm{O}(4)$, from the proper lorentzian models, based on $\mathrm{SL}(2, \mathbb{C})$. A detailed analysis traces this behaviour to the use of a first order action [17], and supports the idea that the implications of the $\cos ($ ) term should be better understood, rather than corrected; other authors suggest ways to modify the vertex function to get the 'correct' asymptotic behaviour [18] 19].

To make the previous discussion more explicit I consider a specific example in the last section of this note: the evolution of the simplest ( 5 points, a 'pentachoron') triangulation of $S^{3}$ from one time to the next. That results in a slice of spacetime made of 304 simplices, with faces dual to either triangles with two vertices at time 0 and one at time 1, or viceversa. Looking closely at one such face, made of four steps, the question arises: are all tetrahedra crossed space-like? classically, with the simplest uniform assumptions, the answer turns out to be no. I write down what would be the contribution of the face if one took the spinor basis in all cases, and what a reasonable justification for this choice could be.

I have excluded the case of tetrahedra with a light-like normal, because including it would have been a considerable complication, but see the very recent ref.[20].

\section{2 spin-foam in terms of spinors}

I use $\eta_{I J}=(-+++), \epsilon^{0123}=1, a, b, \ldots=1,2,3, \alpha, \beta, \ldots=0,1$. More details about the notation used and various identities for spinors, 4 -vectors and antisymmetric tensors have been collected into appendix A.

\section{1 simplicity and closure.}

I begin considering a 4 -simplex with vertices $(a b c d e)$, bounded by tetrahedra $(a b c d),(a b e c)$, (abde), (aced), (bcde), with flat metric $\eta_{I J}$. I shall label the triangles of the tetrahedron $(a b c d)$ : abd, bac, $c a d, d b c$ as $t, s, \ldots$ The triangle $t=(a b d)$ is shared by $(a b c d)$ and (abde) and has an antisymmetric area tensor $S_{t}^{I J}$, with Hodge dual ${ }^{*} S_{t}^{I J}:=\frac{1}{2} \epsilon^{I J K L} S_{t K L}$, $(\text { area })^{2}=\frac{1}{2} S_{t}^{I J} S_{t I J}$.

The components of any antisymmetric tensor, in particular of the generators of $\operatorname{SL}(2, \mathbb{C})$ $J^{I J}$, can be organized as two 3 -vectors $L_{a}$ and $K_{a}$, or their complex 'left' and 'right' 
combinations:

$$
L_{a}:=\frac{1}{2} \epsilon_{a b c} J^{b c}, K_{a}:=J^{0 a} ; \quad J_{a}^{L, R}:=\frac{1}{2}\left(L_{a} \pm i K_{a}\right),
$$

or separating the 'selfdual' and 'antiselfdual' components:

$$
\begin{aligned}
{ }^{*} J^{I J}: & =\frac{1}{2} \epsilon^{I J K L} J_{K L} ; \quad J_{ \pm}^{I J}:=\frac{1}{2}\left(J^{I J} \mp i^{*} J^{I J}\right): \frac{1}{2} \epsilon^{I J K L} J_{ \pm K L}= \pm i J_{ \pm}^{I J} ; \\
\frac{1}{2} \epsilon_{a b c} J_{ \pm}^{b c} & = \pm i J_{ \pm}^{0 a}=J_{a}^{L, R} ; \quad J_{ \pm}^{I J} J_{ \pm I J}=4 J_{a}^{L, R} J_{a}^{L, R}, J_{ \pm}^{I J} J_{\mp I J}=0 .
\end{aligned}
$$

In terms of self-dual and antiself-dual components $J_{t}^{I J}$ becomes:

$$
J_{t}^{I J}=\frac{-1}{\sin \frac{\theta}{2}}\left(e^{i \frac{\theta}{2}} S_{t-}^{I J}+e^{-i \frac{\theta}{2}} S_{t+}^{I J}\right),
$$

and viceversa

$$
S_{t}^{I J}=-\sin \frac{\theta}{2}\left(e^{-i \frac{\theta}{2}} J_{t-}^{I J}+e^{i \frac{\theta}{2}} J_{t+}^{I J}\right) ; \quad{ }^{*} S_{t}^{I J}=i \sin \frac{\theta}{2}\left(e^{-i \frac{\theta}{2}} J_{t-}^{I J}-e^{i \frac{\theta}{2}} J_{t+}^{I J}\right)
$$

Tetrahedra being closed polyhedra, for each tetrahedron $\tau$ the area tensors must satisfy a closure constraint:

$$
\sum_{t \in \tau} S_{t}^{I J}=0
$$

For each pair $t, s$ of triangles of the tetrahedron $(a b c d)$ the quadratic 'diagonal' and 'crossdiagonal' 'simplicity constraints' are expressed as:

$$
\begin{aligned}
& \text { 'diagonal' } \quad S_{t}^{I J *} S_{t I J}=i \sin ^{2} \frac{\theta}{2}\left(e^{i \theta} J_{t a}^{L} J_{t a}^{L}-e^{-i \theta} J_{t a}^{R} J_{t a}^{R}\right)=0, \quad \forall t \\
& \text { 'cross - diagonal' } \quad S_{t}^{I J *} S_{s I J}=i \sin ^{2} \frac{\theta}{2}\left(e^{i \theta} J_{t a}^{L} J_{s a}^{L}-e^{-i \theta} J_{t a}^{R} J_{s a}^{R}\right)=0, \quad \forall t, s
\end{aligned}
$$

they are necessary and sufficient to guarantee that each $S_{t}^{I J}$, and therefore each ${ }^{*} S_{t}^{I J}$, can be written as the antisymmetrized product of two 4-vectors. A solution to these constraints is still a solution if we change $e^{i \theta} \rightarrow-e^{i \theta}$; this corresponds to switching $\gamma \rightarrow-\frac{1}{\gamma}$.

A simpler, linear condition for simplicity is the existence, for each tetrahedron, of a 4 -vector $V^{I}$, perpendicular to all its faces. The proof is simple if $V_{\tau}^{2} \neq 0$ : from the identity

$$
{ }^{*} S_{t}^{I J} V_{\tau}^{M}+{ }^{*} S_{t}^{J M} V_{\tau}^{I}+{ }^{*} S_{t}^{M I} V_{\tau}^{J}=-\epsilon^{I J M N} S_{t N K} V_{\tau}^{K}
$$

since $V_{\tau}^{2} \neq 0$ and $V_{\tau M} S_{t}^{M I}=0$, it follows that:

$$
{ }^{*} S_{t}^{I J}=V^{I} \frac{V_{M}{ }^{*} S_{t}^{M J}}{V^{2}}-\frac{V_{M}{ }^{*} S_{t}^{M I}}{V^{2}} V^{J}:=V^{I} N_{t}^{J}-V^{J} N_{t}^{I}
$$

and the dual relation $S_{t}^{I J}=\epsilon^{I J K L} N_{t K} V_{\tau L}$, with $N_{t}^{I}$ ortogonal to $V_{\tau}^{I}$ and to $S_{t}^{I J}$. So if $V^{I}$ exists, ${ }^{*} S_{t}^{I J}$ is simple, and therefore so is $S_{t}^{I J}$. $V^{I}$ necessarily exists for each tetrahedron, because if for triangles $s, t: S_{t}^{I J}=a^{[I} b^{J]}, S_{s}^{I J}=a^{[I} c^{J]}$, then $V^{I}=\epsilon^{I J K L} a_{J} b_{K} c_{L}$ satisfies the assumptions.

From the definition follows that for any triangle

$$
(\text { area })^{2}=\frac{1}{2} S_{t}^{I J} S_{t I J}=-\frac{1}{4} V_{\tau}^{2} N_{t}^{2}
$$


This is negative for a time-like triangle, that can be brought to lie on the t-z plane, and then we can choose its normals to be either on the $\mathrm{x}$ or on the $\mathrm{y}$ axis; positive for a space-like triangle, that can be brought to lie on the $\mathrm{x}-\mathrm{y}$ plane, and then we can choose its normals to be either on the $\mathrm{t}$ or the $\mathrm{z}$ axis, and $V_{\tau}^{2}$ and $N_{t}^{2}$ will have opposite signs. I shall only deal with this last case, for reasons that will be explained.

One can also show that $S_{(a b d)}^{I J}=k \epsilon^{I J}{ }_{K L} V_{a b c d}^{K} V_{a b d e}^{L}$, or a similar expression in terms of the 4 -vectors $N_{t}^{I}, N_{t}^{\prime I}$ in the two neighbouring tetrahedra.

The conclusion is that for each tetrahedron the constraints can be summarized as:

$$
\begin{gathered}
\text { 'closure' }: \sum_{t} N_{t}^{I}=\sum_{t} \frac{V_{M}{ }^{*} S_{t}^{M I}}{V^{J} V_{J}}=-\frac{i \sin \frac{\theta}{2}}{V^{J} V_{J}} \sum_{t}\left(e^{i \frac{\theta}{2}} V_{M} J_{t+}^{M I}-e^{-i \frac{\theta}{2}} V_{M} J_{t-}^{M I}\right)=0 \\
\text { 'simplicity' }: V_{M} S_{t}^{M I}=-\sin \frac{\theta}{2}\left(e^{i \frac{\theta}{2}} V_{M} J_{t+}^{M I}+e^{-i \frac{\theta}{2}} V_{M} J_{t-}^{M I}\right)=0 . \quad \forall t
\end{gathered}
$$

For space-like or time-like tetrahedra we can choose frames which give simple explicit versions of (2.11):

$$
\begin{array}{ll}
V^{I}=\left(V^{0}, 0,0,0\right): & L_{1}+\frac{1}{\gamma} K_{1}=L_{2}+\frac{1}{\gamma} K_{2}=L_{3}+\frac{1}{\gamma} K_{3}=0 \\
V^{I}=\left(0,0,0, V^{3}\right): & L_{1}-\gamma K_{1}=L_{2}-\gamma K_{2}=L_{3}+\frac{1}{\gamma} K_{3}=0
\end{array}
$$

From 2.10, 2.11 combined we get a simple form of the closure constraints for each tetrahedron, which do not depend on $V^{I}$ :

$$
\sum_{t \in \tau} J_{t a}^{R}=0
$$

\section{$2.2 \quad$ Using spinors}

Following [3], I shall use as basic variables for spin-foam theory a $\mathrm{SL}(2, \mathbb{C})$ group element for each face, and a pair of spinors $\left(u_{t}, t_{t}\right)$ for each triangle. For $\operatorname{SL}(2, \mathbb{C})$ transformations $u$ and $t$ transform as 2 :

$$
u \rightarrow u g \quad t \rightarrow t g^{\dagger-1}
$$

The idea is to implement these transformations through the $\operatorname{SL}(2, \mathbb{C})$ invariant Poisson brackets $\left\{t_{\alpha}, \bar{u}_{\beta}\right\}=-i \delta_{\alpha \beta} ; \quad\left\{u_{\beta}, \bar{t}_{\alpha}\right\}=-i \delta_{\alpha \beta}$ and the generators:

$$
J_{a}^{L}=-\frac{1}{2} u \sigma_{a} \bar{t}, \quad J_{a}^{R}=-\frac{1}{2} t \sigma_{a} \bar{u} ; \quad\left\{J_{a}^{L, R}, J_{b}^{L, R}\right\}=\epsilon_{a b c} J_{c}^{L, R}, \quad\left\{J_{a}^{L}, J_{b}^{R}\right\}=0 .
$$

so that

$$
\left\{u_{\alpha}, J_{a}^{L}\right\}=\frac{i}{2} u_{\beta} \sigma_{a \beta \alpha} ; \quad\left\{t_{\alpha}, J_{a}^{R}\right\}=\frac{i}{2} t_{\beta} \sigma_{a \beta \alpha}
$$

Altogether, with a pair of spinors $\left(t_{t}, u_{t}\right)$ for each of the 4 triangles of a tetrahedron we define $\mathbf{L}_{t}, \mathbf{K}_{t}, \mathbf{J}_{t}^{L, R}$, to which we want to impose the closure and simplicity constraints.

The closure constraints give for each tetrahedron $\tau$, from $(2.13)$ :

$$
\sum_{t \in \tau} \bar{u}_{t \alpha} \sigma_{a \alpha \beta} t_{t \beta}=0
$$

\footnotetext{
${ }^{2}$ since I have $\mathrm{SL}(2, \mathbb{C})$ transformations acting on the right, many of the definitions that follow will be different in detail from [3].
} 
The matrix $\sum_{t \in \tau} \bar{u}_{t \alpha} t_{t \beta}$ must therefore be proportional to the unit matrix, or zero, hence:

$$
\sum_{t \in \tau} \bar{u}_{t \alpha} t_{t \beta}=\frac{1}{2} \sum_{t \in \tau}\left(u_{t}^{\dagger} t_{t}\right) \delta_{\alpha \beta}:=C_{\tau} e^{i \psi_{\tau}} \delta_{\alpha \beta},
$$

with $C_{\tau}$ a real constant. We shall see shortly that for all tetrahedra $e^{i \psi_{\tau}}=e^{i \frac{\theta}{2}}$.

The relations (2.11) expressing the simplicity constraints, multiplied by $\widetilde{\sigma}_{I}=\left(1, \sigma_{a}\right)$ and dropping overall constants become, using (.33, 2.15):

$$
e^{i \frac{\theta}{2}} \widetilde{\sigma}_{I} V^{I} \sigma_{a}\left(u_{t} \sigma_{a} \bar{t}_{t}\right)-e^{-i \frac{\theta}{2}} \sigma_{a}\left(t_{t} \sigma_{a} \bar{u}_{t}\right) \widetilde{\sigma}_{I} V^{I}=0,
$$

where

$$
\sigma_{a}\left(u \sigma_{a} \bar{t}\right)=\left(\begin{array}{cc}
u_{0} \bar{t}_{0}-u_{1} \bar{t}_{1} & 2 u_{1} \bar{t}_{0} \\
2 u_{0} \bar{t}_{1} & u_{1} \bar{t}_{1}-u_{0} \bar{t}_{0}
\end{array}\right)
$$

For either spacelike or timelike tetrahedra we can go to a standard frame in which

$$
V_{0}^{I}=(\alpha, 0,0, \beta)
$$

excluding the light-like case $\alpha^{2}=\beta^{2}$. Indicating the spinors in this frame with capital letters, from 2.18) we get the independent equations:

$$
(\alpha+\beta) e^{i \frac{\theta}{2}} U_{1} \bar{T}_{0}=e^{-i \frac{\theta}{2}}(\alpha-\beta) T_{1} \bar{U}_{0} ; \quad e^{i \frac{\theta}{2}}\left(U_{0} \bar{T}_{0}-U_{1} \bar{T}_{1}\right)=e^{-i \frac{\theta}{2}} T_{0} \bar{U}_{0}-T_{1} \bar{U}_{1} .
$$

in fact, three independent real equations. One can use them to get an explicit relation between the $U$ and the $T$ spinors for each triangle. In fact these equations are satisfied if we set:

$$
T_{0}=\kappa(\beta-\alpha) e^{i \frac{\theta}{2}} U_{0}, T_{1}=\kappa(\beta+\alpha) e^{i \frac{\theta}{2}} U_{1} \rightarrow T_{\alpha}=\kappa e^{i \frac{\theta}{2}} U_{\beta} \sigma_{I \beta \alpha} V_{0}^{I}
$$

where $\kappa$ could be any real number, but for simpliciy I choose $\kappa= \pm 1$. We can go back to an arbitrary frame by boosting $\left(V_{0}^{I}, T_{t}, U_{t}\right)$ to $\left(V^{I}, t_{t}, u_{t}\right)$ with some $g \in S L(2, \mathbb{C})$. From (.31), 2.14 we have:

$$
T_{\alpha}=t_{\beta} g_{\beta \alpha}^{\dagger-1} ; \quad U_{\alpha}=u_{\beta} g_{\beta \alpha} ; \quad V_{0}^{I}=V^{J} \Lambda_{J}^{I}
$$

and the relation between these 'boosted' spinors will be:

$$
t_{t \alpha}-\kappa_{t} e^{i \frac{\theta}{2}} u_{t \beta} \sigma_{I \beta \alpha} V^{I}=0 .
$$

They will 'solve' the constraint eq.s (2.18), because these equations are covariant. Eq. (2.23) is the main result of this section; it is interesting to notice that apart from factors it is the 'twistor equation' of R. Penrose.

The first consequence we want to draw from 2.23 comes from looking at the normals to the triangles $(2.9)$, for which, if the $(2.18)$ are satisfied, one finds:

$$
\widetilde{\sigma}_{I} N_{t}^{I}=\frac{\widetilde{\sigma}_{I} V_{\tau M}{ }^{*} S_{t}^{M I}}{V_{\tau}^{2}}=-\frac{\sin \frac{\theta}{2}}{2 V_{\tau}^{2}}\left(e^{i \frac{\theta}{2}} \widetilde{\sigma}_{I} V_{\tau}^{I} \sigma_{a}\left(u_{t} \sigma_{a} \bar{t}_{t}\right)+e^{-i \frac{\theta}{2}}\left(t_{t} \sigma_{a} \bar{u}_{t}\right) \sigma_{a} \widetilde{\sigma}_{I} V_{\tau}^{I}\right)
$$

Using (2.23) this gives, after some algebra, the remarkably simple expressions:

$$
N_{t}^{I}=\frac{\kappa_{t} \sin \frac{\theta}{2}}{V_{\tau}^{2}}\left(V_{\tau}^{I} V_{\tau}^{J}-\eta^{I J} V_{\tau}^{2}\right) u_{t} \sigma_{J} \bar{u}_{t} ; \quad N_{t}^{2}=-\frac{\sin ^{2} \frac{\theta}{2}}{V_{\tau}^{2}}\left(u_{t} \sigma_{I} V_{\tau}^{I} \bar{u}_{t}\right)^{2}
$$


In two particular cases we have:

$$
\begin{aligned}
& \text { for } V_{\tau}^{I}=\left(V^{0}, 0,0,0\right), N_{t}^{I}=\kappa_{t} \sin \frac{\theta}{2}\left(0,-\left(u_{t} \sigma_{a} \bar{u}_{t}\right)\right) \\
& \text { for } V_{\tau}^{I}=(0, \mathbf{v}), N_{t}^{I}=\kappa_{t} \sin \frac{\theta}{2}\left(-u_{t} \bar{u}_{t}, \frac{1}{\mathbf{v}^{2}}\left(v_{a} v_{b}-\mathbf{v}^{2} \delta_{a b}\right)\left(u_{t} \sigma_{b} \bar{u}_{t}\right)\right) .
\end{aligned}
$$

In general, from the definition of $N_{t}^{I}$ we get:

$$
S_{t}^{I J}=\epsilon^{I J K L} N_{t K} V_{\tau L}=k_{t} \sin \frac{\theta}{2} \epsilon^{I J K L} V_{\tau K} u_{t} \sigma_{L} \bar{u}_{t}
$$

it follows that the 4 -vector $P_{t}^{I}:=u_{t} \sigma^{I} \bar{u}_{t}$, which is null, is orthogonal to $S_{t}^{I J}$ :

$$
S_{t}^{I J} u_{t} \sigma_{I} \bar{u}_{t}=S_{t}^{I J} P_{t I}=0 \text {. }
$$

and must therefore be in the plane spanned by $V_{\tau}^{I}$ and $N_{t}^{I}$; but this implies that $V_{\tau}^{2}$ and $N_{t}^{2}$ have opposite signs, and indeed from 2.25 we see that $V_{\tau}^{2} N_{t}^{2}$ is always negative, and therefore (area) $)^{2}=\frac{1}{2} S_{t}^{I J} S_{t I J}>0$. I would conclude that a configuration in which this does not happen cannot be described by spinors the way we have introduced them.

From the expression of $N_{t}^{I}$ for $V_{\tau}^{I}=(0, \mathbf{v})$ we see that for time-like tetrahedra the $\kappa_{t}$ cannot be all of the same sign, because this would clash with the closure constraint $\sum_{t \in \tau} N_{t}^{0}=0$.

Combining 2.17) with 2.23 we find that if $C_{\tau} \neq 0$ :

$$
\left(\sum_{t \in \tau} \bar{u}_{t \alpha} \kappa_{t} e^{i \frac{\theta}{2}} u_{\gamma}\right) \sigma_{I \gamma \beta} V_{\tau}^{I}=C_{\tau} e^{i \frac{\theta}{2}} \delta_{\alpha \beta}
$$

In this way we find the inverse of $\sigma_{I} V_{\tau}^{I}$; provided that $V_{\tau}^{2} \neq 0$ and $C_{\tau} \neq 0$ we can write the 4 -vector normal to the tetrahedron as

$$
V_{\tau}^{I}=\frac{V_{\tau}^{2}}{2 C_{\tau}} \sum_{t \in \tau} \kappa_{t} u_{t} \sigma^{I} \bar{u}_{t}=\frac{V_{\tau}^{2}}{2 C_{\tau}} \sum_{t \in \tau} \kappa_{t} P_{t}^{I}
$$

Finally from (2.23), for any pair of triangles $t, s$ belonging to a tetrahedron with normal $V_{\tau}^{I}$ and spinors $\left(u_{s}, t_{s}\right),\left(u_{t}, t_{t}\right)$, we can derive, using $\sigma_{I} V^{I} \widetilde{\sigma}_{J} V^{J}=V^{2}$ and the identities in $(.31)$ :

$$
\begin{aligned}
t_{t \alpha} \epsilon_{\alpha \beta} t_{s \beta} & +\kappa_{s} \kappa_{t} V_{\tau}^{2} e^{i \theta} u_{t \alpha} \epsilon_{\alpha \beta} u_{s \beta}=0 ; \\
t_{t \alpha} \bar{u}_{t \alpha} & =\kappa_{t} e^{i \frac{\theta}{2}} u_{t \alpha} \sigma_{I \alpha \beta} V^{I} \bar{u}_{t \beta}=e^{i \theta} \bar{t}_{t \alpha} u_{t \alpha}
\end{aligned}
$$

eq. 2.31 implies $e^{i \psi_{\tau}}=e^{i \frac{\theta}{2}}$, as anticipated.

Eq.s 2.30) are the 'holomorphic simplicity constraints' of [3]; they form a set of first class constraints, but one can see that they imply (2.6) and the second class $(2.7)^{3}$; in fact, from the definition 2.15 , using .30 we have:

$\mathbf{J}_{t}^{L} \cdot \mathbf{J}_{s}^{L}=\frac{1}{4} t_{t}^{\dagger} u_{t} t_{s}^{\dagger} u_{s}-\frac{1}{2} \bar{t}_{t \alpha} \epsilon_{\alpha \gamma} \bar{t}_{s \gamma} u_{t \beta} \epsilon_{\beta \delta} u_{s \delta}, \quad \mathbf{J}_{t}^{R} \cdot \mathbf{J}_{s}^{R}=\frac{1}{4} u_{t}^{\dagger} t_{t} u_{s}^{\dagger} t_{s}-\frac{1}{2} \bar{u}_{t \alpha} \epsilon_{\alpha \gamma} \bar{u}_{s \gamma} t_{t \beta} \epsilon_{\beta \delta} t_{s \delta}$.

and from 2.30$)$ :

$$
e^{i \theta} t_{t}^{\dagger} u_{t} t_{s}^{\dagger} u_{s}=e^{-i \theta} u_{t}^{\dagger} t_{t} u_{s}^{\dagger} t_{s} ; \quad e^{i \theta} \bar{t}_{t \alpha} \epsilon_{\alpha \beta} \bar{t}_{s \beta} u_{t \gamma} \epsilon_{\gamma \delta} u_{s \delta}=e^{-i \theta} t_{t \alpha} \epsilon_{\alpha \beta} t_{s \beta} \bar{u}_{t \gamma} \epsilon_{\gamma \delta} \bar{u}_{s \delta},
$$

from which (2.6) (2.7) follow.

Overall, in this scheme the triangle $t=(a b d)$ is shared by $(a b c d)$ and (abde) within a 4-simplex (abcde); these tetrahedra have different normal vectors $V_{a b c d}^{I}, V_{a b d e}^{I}$ and the triangle different normals, but the same $S_{t}^{I J}$ and spinors $\left(u_{t}, t_{t}\right)$.

\footnotetext{
${ }^{3}$ notice that $(2.23)$ and 2.30 do not Poisson-commute with their complex conjugates
} 


\section{The quantum theory}

\subsection{Representations of $\mathrm{SL}(2, \mathbb{C})$}

In the quantum theory the constraints should be imposed by restricting the sum/integral over the representations of $\mathrm{SL}(2, \mathbb{C})$, an idea that goes back to the orignal formulation of spin-foam theory[1], although the breakthrough in the theory came much later, with the EPRL [4] idea that this restriction should be derived imposing the constraints on the states, à la Gupta-Bleurer 4

Given a triangulation, the contribution of a face to the transition amplitude is determined by a sequence of transitions between 'states'. These states are elements of $\mathcal{H}^{(n, \rho)}$, the Hlbert space on which a unitary representation of $\operatorname{SL}(2, \mathbb{C})$ acts; they encode the geometry of the tetrahedron involved, and must be such that the expectation of $J^{I J}$ satisfies the simplicity constraints.

The theory of unitary representations of $\operatorname{SL}(2, \mathbb{C})[14$ is the work of I. M. Gel'fand and his collaborators; the representations are infinite dimensional, labeled by the two indices $(n, \rho)$ with $n$ integer, $\rho$ real $\in(-\infty, \infty)$. On functions of a spinor $u=\left(u_{0} u_{1}\right)$ and its c.c., homogeneous of degree $(\lambda, \mu)=\left(\frac{n}{2}+i \frac{\rho}{2}-1,-\frac{n}{2}+i \frac{\rho}{2}-1\right)$, they act as $T_{g}^{(n \rho)} f(u, \bar{u})=f(u \cdot g, \bar{u} \cdot \bar{g})$. With the measure $\Omega_{u}=\frac{i}{2}\left(u_{0} d u_{1}-u_{1} d u_{0}\right) \wedge\left(\bar{u}_{0} d \bar{u}_{1}-\bar{u}_{1} d \bar{u}_{0}\right)$ these functions form the Hilbert space $\mathcal{H}^{(n, \rho)}$. There are however other realizations of $\mathcal{H}^{(n, \rho)}$, as we shall see in a moment.

A simple version of Gelfand's construction has been given by Smorodinskii and Huszar [21] 22, based on the idea of separating right and left generators of $\operatorname{SL}(2, \mathbb{C})$, extending the theory of representations of SU(2) with $J_{3}^{L}, J_{3}^{R}$ diagonal; the unitarity of the representation requires $\left(J_{a}^{L}\right)^{\dagger}=J_{a}^{R}$. By simple algebraic manipulations $5^{5}$ they find that the Casimir operators for the $(n, \rho)$ representation can be expressed as:

$$
\left(\mathbf{J}^{L}\right)^{2}=\frac{1}{4}\left(\frac{n}{2}-i \frac{\rho}{2}\right)^{2}-\frac{1}{4} ; \quad\left(\mathbf{J}^{R}\right)^{2}=\frac{1}{4}\left(\frac{n}{2}+i \frac{\rho}{2}\right)^{2}-\frac{1}{4}
$$

equivalent to the more common:

$$
C_{1}=J^{I J} J_{I J}=2\left(\mathbf{L}^{2}-\mathbf{K}^{2}\right)=\frac{1}{2}\left(n^{2}-\rho^{2}-4\right), \quad C_{2}=J^{I J *} J_{I J}=-4 \mathbf{L} \cdot \mathbf{K}=n \rho
$$

These expressions immediately suggest that in a quantum theory, for large $n$ and ignoring the $\frac{1}{4}$, the constraint 2.6 will be satified if we limit the sum over representations of $\mathrm{SL}(2, \mathbb{C})$ to those such that:

$$
e^{i \theta}\left(\mathbf{J}^{L}\right)^{2}=e^{-i \theta}\left(\mathbf{J}^{R}\right)^{2} \rightarrow \frac{(n+i \rho)^{2}}{(n-i \rho)^{2}}=e^{2 i \theta}=\frac{(1+i \gamma)^{2}}{(1-i \gamma)^{2}}
$$

As already pointed out after eq.s 2.6 2.7), this equation has two solutions: $\rho=n \gamma$, or $\rho=-\frac{n}{\gamma}$, that correspond to the two choices $\pm e^{i \theta}$ for the square root of $e^{2 i \theta}$. But now we can see that positive $(\text { area })^{2}$, the only case we want to deal with, correspond to the choice

4 [9] has a particularly lucid explanation.

5 and demanding that the group representation be one valued 
$\rho=\gamma n$; In fact, with some algebra, I find that 2.4 and $(3.3)$ imply that:

$$
\begin{gathered}
(\text { area })^{2}=\frac{1}{2} S^{I J} S_{I J}=\frac{\gamma^{2}}{2\left(1+\gamma^{2}\right)^{2}}\left(\left(1-\gamma^{2}\right) C_{1}+2 \gamma C_{2}\right) \\
\left.\rho=\gamma n \rightarrow(\text { area })^{2}=\frac{1}{4} \gamma^{2} n^{2}-\frac{\gamma^{2}\left(1-\gamma^{2}\right)}{2\left(1+\gamma^{2}\right)^{2}} ; \quad \rho=-\frac{n}{\gamma} \rightarrow(\text { area })^{2}=-\frac{1}{4} n^{2}-\frac{\gamma^{2}\left(1-\gamma_{3}^{2}\right)}{\left(1+\gamma^{2}\right)^{2}} 4\right)
\end{gathered}
$$

Therefore choosing $\rho=\gamma n$ one has that, up to terms which do not grow with $n$, (area $)^{2}$ is positive and quantized, one of the key results of loop quantum gravity.

With the same algebra ${ }^{6}$ that leads to $(3.1)$ one defines a 'canonical basis' for the Hilbert space $\mathcal{H}^{(n, \rho)}$; it consists of eigenstates $\psi_{m}^{j}$ of $\left(\mathbf{L}^{2}, L_{3}\right)$ 'injected' in $\mathcal{H}^{(n, \rho)}$, such that in particular:

$$
\begin{aligned}
& \mathbf{L}^{2} \psi_{m}^{(n, \rho) j}=j(j+1) \psi_{m}^{(n, \rho) j} ; \quad L_{3} \psi_{m}^{(n, \rho) j}=m \psi_{m}^{(n, \rho) j} ; \quad L_{ \pm} \psi_{m}^{(n, \rho) j}=\sqrt{(j \pm m+1)(j \mp m)} \psi_{m \pm 1}^{(n, \rho) j} \\
& K_{3}\left|\psi_{m}^{(n, \rho) j}>=C_{j}\right| \psi_{m}^{(n, \rho) j-1}>-\frac{\rho n m}{4 j(j+1)}\left|\psi_{m}^{(n, \rho) j}>-C_{j+1}\right| \psi_{m}^{(n, \rho) j+1}>.
\end{aligned}
$$

(the coefficients $C_{j}$ depend on $\rho, n, m$ besides $j$; we shall not need their form).

I shall use a realization of $\mathcal{H}^{(n, \rho)}$ as a space of functions on light-like 4-vectors $P^{I}$ [29] [22] [14], derived from a spinor $u_{\alpha}$ by $P^{I}:=u \sigma^{I} \bar{u}$. This choice is in principle convenient because for any triangle $t$ we have a null 4 -vector $P_{t}^{I}=u_{t} \sigma^{I} \bar{u}_{t}$, and we have seen earlier 2.27) that the simplicity constraints imply that $P_{t}^{I}$ is orthogonal to $S_{t}^{I J}$. To get an element of $\mathcal{H}^{(n, \rho)}$ we start from a function $\Phi(P)$ such that $\Phi\left(e^{\alpha} P\right)=e^{\left(i \frac{\rho}{2}-1\right) \alpha} \Phi(P)$, and set

$$
f\left(u_{0}, u_{1}\right)=\left(\frac{u_{0}}{\bar{u}_{0}}\right)^{\frac{n}{2}} \Phi(P)
$$

From this definition follows that under $\mathrm{SL}(2, \mathbb{C})$ transformations, from .31 :

$$
T_{g}^{(n \rho)}\left(\left(\frac{u_{0}}{\bar{u}_{0}}\right)^{\frac{n}{2}} \Phi\left(u \sigma^{I} \bar{u}\right)\right)=\left(\frac{(u g)_{0}}{(\bar{u} \bar{g})_{0}}\right)^{\frac{n}{2}} \Phi\left(u g \sigma^{I} g^{\dagger} \bar{u}\right)=\left(\frac{(u g)_{0}}{(\bar{u} \bar{g})_{0}}\right)^{\frac{n}{2}} \Phi\left(P^{J} \Lambda_{J}^{I}\right)
$$

The natural invariant scalar product would be

$$
<\Psi \mid \Phi>=\int \theta\left(P^{0}\right) \delta\left(P^{2}\right) \frac{d^{4} P}{(2 \pi)^{3}} \overline{\Psi(P)} \Phi(P)=\int \frac{d^{3} P}{2(2 \pi)^{3} P} \overline{\Psi(P)} \Phi(P)
$$

For infinitesimal transformations $g(\epsilon, \eta)=1+\frac{1}{2}\left(i \epsilon_{a}+\eta_{a}\right) \sigma_{a} \simeq\left(1+i \epsilon_{a} L_{a}+i \eta_{a} K_{a}\right)$ :

$$
\begin{aligned}
T_{g(\epsilon, \eta)}^{(n \rho)}\left(\left(\frac{u_{0}}{\bar{u}_{0}}\right)^{\frac{n}{2}} \Phi\left(u \sigma^{I} \bar{u}\right)\right)= & \left(\frac{u_{0}+\frac{1}{2} u_{0}\left(i \epsilon_{3}+\eta_{3}\right)+\frac{1}{2} u_{1}\left(i \epsilon_{1}+\eta_{1}-\epsilon_{2}+i \eta_{2}\right)}{\bar{u}_{0}+\frac{1}{2} \bar{u}_{0}\left(-i \epsilon_{3}+\eta_{3}\right)+\frac{1}{2} \bar{u}_{1}\left(-i \epsilon_{1}+\eta_{1}-\epsilon_{2}-i \eta_{2}\right)}\right)^{\frac{n}{2}} . \\
& \cdot \Phi\left(u_{\alpha} \sigma_{\alpha \beta}^{I} \bar{u}_{\beta}+\frac{i}{2} \epsilon_{a} u\left[\sigma_{a}, \sigma^{I}\right] \bar{u}+\frac{1}{2} \eta_{a} u\left\{\sigma_{a}, \sigma^{I}\right\} u\right)= \\
= & \left(\frac{u_{0}}{\bar{u}_{0}}\right)^{\frac{n}{2}}\left(1+i \sum\left(\epsilon_{k} L_{k}+\eta_{k} K_{k}\right)\right) \Phi(P) ;
\end{aligned}
$$

expanding, we have for the generators of $\mathrm{SL}(2, \mathbb{C})[29][22]$ :

$$
\begin{aligned}
L_{1} & =-i\left(P_{2} \partial_{3}-P_{3} \partial_{2}\right)+\frac{n P_{1}}{2\left(P+P_{3}\right)}, \quad L_{2}=-i\left(P_{3} \partial_{1}-P_{1} \partial_{3}\right)+\frac{n P_{2}}{2\left(P+P_{3}\right)}, \\
L_{3} & =-i\left(P_{1} \partial_{2}-P_{2} \partial_{1}\right)+\frac{n}{2} \\
K_{1} & =-i P \partial_{1}-\frac{n P_{2}}{2\left(P+P_{3}\right)}, \quad K_{2}=-i P \partial_{2}+\frac{n P_{1}}{2\left(P+P_{3}\right)}, \quad K_{3}=-i P \partial_{3},
\end{aligned}
$$

\footnotetext{
${ }^{6}$ given as problem 2.9 in 23 !
} 
where $P^{0}=P=\sqrt{P_{1}^{2}+P_{2}^{2}+P_{3}^{2}}$, and $\partial_{a} \Phi:=\left.\frac{\partial \Phi}{\partial P_{a}}\right|_{P^{0}}+\left.\frac{\partial \Phi}{\partial P^{0}}\right|_{P_{a}} \frac{\partial P}{\partial P_{a}}$.

If one takes $P^{I}=e^{\alpha}(a, b \cos \varphi, b \sin \varphi, c), a=\sqrt{b^{2}+c^{2}}$, then:

$$
\frac{\partial}{\partial \alpha}=P_{a} \partial_{a} ; \quad \frac{\partial}{\partial \varphi}=P_{1} \partial_{2}-P_{2} \partial_{1} \quad \rightarrow \quad L_{3}=-i \frac{\partial}{\partial \varphi}+\frac{n}{2}
$$

For $\Phi \in \mathcal{H}^{(n, \rho)}, \frac{\partial \Phi}{\partial \alpha}=\left(i \frac{\rho}{2}-1\right) \Phi$; therefore there is no information in the dependance of $\Phi$ on $\alpha$. Apart from a factor $e^{\left(i \frac{\rho}{2}-1\right) \alpha}$ the homogeneous function $\Phi$ is completely determined by its value on the intersection of the light-cone with the sphere $P=e^{\alpha}$, or with the plane $P^{3}=e^{\alpha}$, or with the hyperbola $\left(P^{0}\right)^{2}-\left(P^{3}\right)^{2}=e^{2 \alpha}$. I shall consider three basis adapted to each choice; in each case for the scalar product (3.8) should be multiplied by an appropriate $\delta$ function, or the integration in $d \alpha$ over the generators of the light-cone simply omitted.

\subsection{The various basis of $\mathcal{H}^{(n, \rho)}$.}

To realize explicitely the canonical basis I specialize further the choice of variables choosing:

$$
u=e^{\frac{a}{2}}\left(\cos \frac{\theta}{2} e^{i \frac{\varphi}{2}}, \sin \frac{\theta}{2} e^{-i \frac{\varphi}{2}}\right), \quad P^{I}=e^{a}(1, \sin \theta \cos \varphi, \sin \theta \sin \varphi, \cos \theta) .
$$

To impose the simplicity constraints we use our knowledge of action of $K_{3}$ on the basis states of the canonical basis (3.5). Choosing $V^{I}=\left(V^{0}, 0,0,0\right)$, we find [9] for the component 3 of the constraint 2.12 :

$$
<\psi_{m^{\prime}}^{(n \rho) j}\left|\left(L_{3}+\frac{1}{\gamma} K_{3}\right)\right| \psi_{m}^{(n \rho) j}>=\delta_{m m^{\prime}}\left(m-\frac{\rho n m}{4 j(j+1) \gamma}\right)
$$

If $\rho=\gamma n$, this will be approximately 0 if we take $j=\frac{n}{2}$. Then the expectation values of components 1 and 2 will also vanish, because

$$
L_{ \pm}+\frac{1}{\gamma} K_{ \pm}= \pm\left[L_{3}+\frac{1}{\gamma} K_{3}, L_{ \pm}\right]
$$

where $L_{ \pm}=L_{1} \pm i L_{2}, K_{ \pm}=K_{1} \pm i K_{2}$. This $\rho=n \gamma, j=\frac{n}{2}$ solution on the other hand matches very nicely with the quantization of areas in loop quantum gravity. In fact, since $\rho=\gamma n$, the constraint implies:

$$
\begin{aligned}
\left(\mathbf{L}+\frac{1}{\gamma} \mathbf{K}\right)^{2} & =\left(1+\gamma^{2}\right) \mathbf{L}^{2}-\frac{1}{2} C_{1}-\frac{1}{2} \gamma C_{2}=\left(1+\gamma^{2}\right)\left(\mathbf{L}^{2}-\frac{1}{4} n^{2}\right)+1 \simeq 0 \\
& \rightarrow\left(\text { area }_{\mathrm{t}}\right)^{2}=\gamma^{2} \mathbf{L}^{2}=\gamma^{2} j(j+1)
\end{aligned}
$$

To find the corresponding basis functions I use the expresson of $\mathbf{L}^{2}$ in these coordinates and a property of the Wigner functions:

$$
\begin{gathered}
\mathbf{L}^{2}=-\frac{1}{\sin \theta} \frac{\partial}{\partial \theta} \sin \theta \frac{\partial}{\partial \theta}-\frac{1}{\sin ^{2} \theta} \frac{\partial^{2}}{\partial \theta^{2}}+\frac{n}{1+\cos \theta} \\
\left(-\frac{1}{\sin \theta} \frac{\partial}{\partial \theta} \sin \theta \frac{\partial}{\partial \theta}+\frac{1}{\sin ^{2} \theta}\left(m^{2}+m^{\prime 2}-2 m m^{\prime} \cos \theta\right)\right) d_{m m^{\prime}}^{j}(\theta)=j(j+1) d_{m m^{\prime}}^{j}
\end{gathered}
$$


One finds [22] the basis functions that diagonalize $\left(\mathbf{L}^{2}, L_{3}\right)$ :

$$
\begin{array}{r}
\mid \psi_{m}^{(n \rho) \frac{n}{2}}>\leftrightarrow \psi_{m}^{(n \rho) \frac{n}{2}}(a, \theta, \varphi)=C_{a} e^{\left(i \frac{\rho}{2}-1\right) a} e^{i\left(m-\frac{n}{2}\right) \varphi} d_{m \frac{n}{2}}^{\frac{n}{2}}(\theta), \\
C_{a}=2 \pi \sqrt{n+1} ; \quad-\frac{n}{2} \leq m \leq \frac{n}{2} ; \quad<\psi_{m^{\prime}}^{(n \rho) \frac{n}{2}} \mid \psi_{m}^{(n \rho) \frac{n}{2}}>=\delta_{m m^{\prime}}
\end{array}
$$

Coherent states carry the information on the geometry of a given triangle, in this case on the normal $\mathbf{n}=\frac{\mathbf{N}}{|\mathbf{N}|}=(\sin \bar{\theta} \cos \bar{\varphi}, \sin \bar{\theta} \sin \bar{\varphi}, \cos \bar{\theta})$; they are defined applying to the 'highest weight' state $\mid \psi_{\frac{n}{2}}^{(n \rho) \frac{n}{2}}>$ the rotation:

$$
\begin{gathered}
u_{\mathbf{n}}=e^{-i \frac{\sigma_{3}}{2} \bar{\varphi}} e^{-i \frac{\sigma_{2}}{2} \bar{\theta}} ; \quad u_{\mathbf{n}}^{\dagger}\left(\begin{array}{l}
\sigma_{1} \\
\sigma_{2} \\
\sigma_{3}
\end{array}\right) u_{\mathbf{n}}=\left(\begin{array}{ccc}
\cos \bar{\theta} \cos \bar{\varphi} & -\sin \bar{\varphi} & \sin \bar{\theta} \cos \bar{\varphi} \\
\cos \bar{\theta} \sin \bar{\varphi} & \cos \bar{\varphi} & \sin \bar{\theta} \sin \bar{\varphi} \\
-\sin \bar{\theta} & 0 & \cos \bar{\theta}
\end{array}\right)\left(\begin{array}{l}
\sigma_{1} \\
\sigma_{2} \\
\sigma_{3}
\end{array}\right) . \\
\left|\psi_{\mathbf{n}}^{(n \rho) \frac{n}{2}}>:=\mathcal{D}^{\frac{n}{2}}\left(u_{\mathbf{n}}\right)\right| \psi_{\frac{n}{2}}^{(n \rho) \frac{n}{2}}>; \quad<\psi_{\mathbf{n}}^{(n \rho) \frac{n}{2}}\left|L_{a}\right| \psi_{\mathbf{n}}^{(n \rho) \frac{n}{2}}>=\frac{n}{2} n_{a}
\end{gathered}
$$

These states are of minimal uncertainty; for example, indicating expectation values on them simply as $<$. $>$ we have

$$
\frac{\Delta L}{<|L|>}=\frac{\sqrt{<\left(L_{a}-<L_{a}>\right)\left(L_{a}-<L_{a}>\right)>}}{\sqrt{<L_{a} L_{a}>}}=\frac{\sqrt{j(j+1)-j^{2}}}{\sqrt{j(j+1)}} \simeq \frac{\sqrt{j}}{j}
$$

For time-like tetrahedra one would choose $V^{I}=(0,0,0, \pm 1)$; its little group is $\mathrm{SU}(1,1)$, the set of all $v \in \mathrm{SL}(2, \mathbb{C})$ such that $v^{\dagger} \sigma_{3} v=\sigma_{3}$. With its unitary irreducible representations [24] one defines a 'pseudobasis' 25] for $\mathcal{H}^{(n, \rho)}$. The reduction of $\mathcal{H}^{(n, \rho)}$ to representations of $\mathrm{SU}(1,1)$ has been studied very thoroughly by $\mathrm{F}$. Conrady and J. Hnybida [9, [10, [26], and before them by many authors, beginning with A. Sciarrino and M. Toller [27] 25] 28]. SU $(1,1)$ has infinite dimensional unitary representations labeled by the eigenvalue of its Casimir operator $Q=L_{3}^{2}-K_{1}^{2}-K_{2}^{2}$, which has a discrete spectrum $q=k(k-1), k$ (half)integer, hence positive $\mathrm{q}$, and a continous one $q=k(k+1), k=-\frac{1}{2}+i s, 0<s<\infty$, hence negative q; eigenstates of $\left(Q, L_{3}\right)$ injected in $\mathcal{H}^{(n, \rho)}$ form altogether the pseudobasis. Choosing variables:

$$
\begin{aligned}
u & =\left(e^{\frac{b}{2}} \cosh \frac{t}{2} e^{i \frac{\varphi}{2}}, e^{\frac{b}{2}} \sinh \frac{t}{2} e^{-i \frac{\varphi}{2}}\right), \quad P^{I}=\left(e^{b} \cosh t, e^{b} \sinh t \cos \varphi, e^{b} \sinh t \sin \varphi, e^{b}\right) \\
Q & =L_{3}^{2}-K_{1}^{2}-K_{2}^{2}=\frac{1}{\sinh t} \frac{\partial}{\partial t} \sinh t \frac{\partial}{\partial t}+\frac{1}{\sinh ^{2} t} \frac{\partial^{2}}{\partial \varphi^{2}}+n \frac{1}{1+\cosh t} L_{3} .
\end{aligned}
$$

We are only concerned with the states in the discrete spectrum, because spinors are suitable only for positive (area $)^{2}$, hence $Q>0$ representation. In fact, using the constraints and $\rho=\gamma n$ as above [9], we find for the quantized areas:

$$
\begin{aligned}
&\left(L_{1}-\gamma K_{1}\right)^{2}+\left(L_{2}-\gamma K_{2}\right)^{2}-\left(K_{3}+\gamma L_{3}\right)^{2}=\frac{1}{2} C_{1}+\frac{1}{2} \gamma C_{2}-\left(1+\gamma^{2}\right) Q= \\
&=\left(1+\gamma^{2}\right)\left(\frac{1}{4} n^{2}-Q\right)-1 \simeq 0 \rightarrow(\text { area })^{2} \simeq \gamma^{2} Q=\gamma^{2} k(k-1)
\end{aligned}
$$

For the discrete series, the analogues of $(3.17,3.5)$ are 28]:

$$
\begin{gathered}
Q\left|\psi_{m}^{(n, \rho) k \pm}>=k(j-1)\right| \psi_{m}^{(n, \rho) k \pm}>; \quad L_{3}\left|\psi_{m}^{(n, \rho) k \pm}>=m\right| \psi_{m}^{(n, \rho) k \pm}>; \\
\left(K_{2} \mp i K_{1}\right)\left|\psi_{m}^{(n \rho) k \pm}>=\sqrt{(m \mp k+1)(m \pm k)}\right| \psi_{m \pm 1}^{(n \rho) k \pm}> \\
K_{3} f_{m}^{(n \rho) k \pm}=A_{m}^{(n \rho) k} f_{m}^{(n \rho)(k+1) \pm}-\frac{\rho m n}{4 k(k-1)}\left|\psi_{m}^{(n \rho) k \pm}>+A_{m}^{(b \rho) k-1}\right| \psi_{m}^{(n \rho)(k-1) \pm}>
\end{gathered}
$$


(we do not need the expression of $\left.A_{m}^{(n \rho) k}\right)$. If $V^{I}=(0,0,0,1)$, the expectation value of component 3 of the constraint $(2.12)$ is given by:

$$
<\psi_{m^{\prime}}^{(n \rho) k \pm}\left|\left(L_{3}+\frac{1}{\gamma} K_{3}\right)\right| \psi_{m}^{(n \rho) k \pm}>=\delta_{m m^{\prime}}\left(m-\frac{\rho n m}{4 k(k-1) \gamma}\right)
$$

which, given that $\rho=\gamma n$, will vanish if $k=\frac{n}{2}$. Then the expectation values of components 1 and 2 will also vanish because:

$$
K_{ \pm}-\frac{1}{\gamma} L_{ \pm}= \pm\left(\left[L_{3}+\frac{1}{\gamma} K_{3}, K_{ \pm}\right]\right)
$$

In conclusion, the states $\mid \psi_{m}^{(n \rho) k \pm}>$ of the discrete spectrum that will contribute are those with $k=\frac{n}{2}$, and $m=k, k+1, \ldots$ or $m=-k,-k-1, \ldots$

The matrix elements of the representations are given in detail in 24] [26]; I quote, for $m^{\prime}>0, m+m^{\prime}>0$, the building bloc:

$$
D_{m^{\prime} m}^{k}\left(e^{i L_{3} \psi} e^{i K_{2} t} e^{i L_{3} \varphi}\right)=e^{i m^{\prime} \psi} b_{m^{\prime} m}^{k}(t) e^{i m \varphi}, \quad b_{m^{\prime} m}^{k}(t)=\sqrt{(-1)^{m-m^{\prime}}} d_{m^{\prime} m}^{k}(i t),
$$

and from [24], the analogue of (3.17):

$$
\left(\frac{1}{\sinh t} \frac{\partial}{\partial t} \sinh t \frac{\partial}{\partial t}-\frac{1}{\sinh ^{2} t}\left(m^{2}+m^{2}-2 m m^{\prime} \cosh t\right)\right) b_{m m^{\prime}}^{k}(t)=k(k-1) b_{m m^{\prime}}^{k}(t)
$$

from which we derive the basis functions that diagonalize $\left(Q, L_{3}\right)$ :

$$
\psi_{m}^{(n \rho) k \pm}=C_{b} e^{\left(i \frac{\rho}{2}-1\right) b} e^{i\left(m-\frac{n}{2}\right) \varphi} b_{m \frac{n}{2}}^{\frac{n}{2}}(t)
$$

Coherent states for the discrete series can be built as:

$$
\begin{gathered}
v_{N}=e^{i \bar{\varphi} L_{3}} e^{-i \bar{t} K_{1}}=e^{i \sigma_{3} \frac{\bar{\varphi}}{2}} e^{\sigma_{1} \frac{\bar{t}}{2}} \in S U(1,1) ; \quad\left|\psi_{N}^{(n \rho) k \pm}>:=\mathcal{D}^{k \pm}\left(v_{N}\right)\right| \psi_{ \pm k}^{(n \rho) k \pm}> \\
<\psi_{N}^{(n \rho) k \pm}\left|\left(K_{1}, K_{2}, L_{3}\right)\right| \psi_{N}^{(n \rho) k \pm}>= \pm k(\sinh \bar{t} \sin \bar{\varphi}, \sinh \bar{t} \cos \bar{\varphi}, \cosh \bar{t})
\end{gathered}
$$

$N$ describes a time-like two-sheeted hyperboloid. For the details on the construction of the pseudobasis and of the coherent states see [9]. So far nobody has taken up the challenge of using it, work out the asymptotic limit etc..

Finally, I want to consider what I regard as the most interesting basis, the one based on the abelian subgroup of $\operatorname{SL}(2, \mathbb{C})$ generated by $\left(L_{3}, K_{3}\right)$; it has been studied in [22] [30] 31]. A suitable parametrization is:

$$
\begin{aligned}
u_{\alpha} & =\left(\frac{1}{\sqrt{2}} e^{\frac{c}{2}} e^{\frac{u+i \varphi}{2}}, \frac{1}{\sqrt{2}} e^{\frac{c}{2}} e^{\frac{-u-i \varphi}{2}}\right), \quad P^{I}=e^{c}(\cosh u, \cos \varphi, \sin \varphi, \sinh u) ; \\
L_{3} & =-i \partial_{\varphi}+\frac{n}{2}, \quad L_{ \pm}=e^{ \pm i \varphi}\left( \pm \sinh u \partial_{c} \mp \cosh u \partial_{u}+i \sinh u \partial_{\varphi}+\frac{n}{2} e^{-u}\right) \\
K_{3} & =-i \partial_{u}, \quad K_{ \pm}=e^{ \pm i \varphi}\left(i \cosh u \partial_{c}-i \sinh u \partial_{u} \mp \cosh u \partial_{\varphi} \mp i \frac{n}{2} e^{-u}\right) \\
\mathbf{L}^{2} & =-(\sinh u)^{2} \partial_{u}^{2}-(\cosh u)^{2} \partial_{\varphi}^{2}-(\cosh u)^{2} \partial_{u}^{2}+\sinh 2 u \partial_{u} \partial_{u}+\partial_{u}+n e^{-u} \cosh u\left(B_{3} 24\right)
\end{aligned}
$$


The eigenfunctions of $\left(L_{3}, K_{3}\right)$ are, with $m$ (half)integer, $\lambda$ real, $-\infty<\lambda<\infty$ :

$$
\begin{aligned}
\psi_{m \lambda}^{(n \rho)}(c, u, \varphi) & =\frac{\sqrt{2}}{2 \pi} e^{\left(i \frac{\rho}{2}-1\right) c} e^{i\left(m-\frac{n}{2}\right) \varphi} e^{i \lambda u} \\
L_{3} \psi_{m \lambda}^{(n \rho)} & =m \psi_{m \lambda}^{(n \rho)} ; \quad K_{3} \psi_{m \lambda}^{(n \rho)}=\lambda \psi_{m \lambda}^{(n \rho)} ; \quad<\psi_{m \lambda}^{(n \rho)} \mid \psi_{m^{\prime} \lambda^{\prime}}^{(n \rho)}>=\delta\left(\lambda-\lambda^{\prime}\right) \delta_{m m^{\prime}} \\
L_{ \pm} \psi_{m \lambda}^{(n \rho)} & =\psi_{m \pm 1, \lambda}^{(n \rho)}\left(\cosh u\left(\frac{n}{2} \pm i \lambda\right)-\sinh u\left(m \mp \frac{\rho}{2} \pm 1\right)\right) \\
K_{ \pm} \psi_{m \lambda}^{(n \rho)} & =\psi_{m \pm 1, \lambda}^{(n \rho)}\left(\cosh u\left(1 \mp i m-\frac{\rho}{2}\right)-\sinh u\left(\lambda \pm i \frac{n}{2}\right)\right)
\end{aligned}
$$

These states have been studied in [21] [22], and have been used in [32] as eigenstates of the 'energy' of a Rindler horizon. To satisfy the simplicity constraints we set $\lambda=\gamma \mathrm{m}$ for the 3-rd component, and let (3.14) (3.21) take care of the other two. There are no highest weight states, and therefore no coherent states as we had them before, so the question is whether we can do without them, and still have states which somehow encode the geometry of the triangles.

Suppose we smear these states, using $f_{\delta}(\lambda):=\frac{1}{(2 \pi)^{1 / 4} \delta^{1 / 2}} e^{-\frac{\lambda^{2}}{4 \delta^{2}}}$, and defining

$$
\left|m \lambda \delta>:=\int d \lambda^{\prime} f_{\delta}\left(\lambda^{\prime}-\lambda\right)\right|(n \rho) m \lambda^{\prime}>\leftrightarrow \psi_{m \lambda \delta}^{(n \rho)}=\frac{(2 \pi)^{1 / 4} \sqrt{\delta}}{\pi} e^{\left(i \frac{\rho}{2}-1\right) c} e^{i\left(m-\frac{n}{2}\right) \varphi} e^{i \lambda u} e^{-u^{2} \delta^{2}}
$$

With this regulariztion we have that:

$$
<m^{\prime} \lambda^{\prime} \delta\left|m \lambda \delta>=\delta_{m m^{\prime}} e^{-\frac{\left(\lambda-\lambda^{\prime}\right)^{2}}{8 \delta^{2}}}, \quad<m \lambda \delta\right|\left(L_{a}+i K_{a}\right) \mid m \lambda \delta>=(0,0, m+i \lambda)
$$

Therefore, if we boost the regularized states with $\mathcal{D}^{(n \rho)}(g)$ or $\mathcal{D}^{(n \rho)}(v)$ we get states such that:

$$
\begin{aligned}
\mid \psi_{\mathbf{n}}^{(n \rho) \frac{n}{2}}>:= & \mathcal{D}^{(n \rho)}\left(u_{\mathbf{n}}\right)\left|m \lambda \delta>; \quad<\psi_{\mathbf{n}}^{(n \rho) \frac{n}{2}}\right| L_{a} \mid \psi_{\mathbf{n}}^{(n \rho) \frac{n}{2}}>=\frac{n}{2} n_{a} \\
\mid \psi_{N}^{(n \rho) k \pm}>:= & \mathcal{D}^{(n \rho)}\left(v_{N}\right) \mid m \lambda \delta>; \\
& <\psi_{N}^{(n \rho) k \pm}\left|\left(K_{1}, K_{2}, L_{3}\right)\right| \psi_{N}^{(n \rho) k \pm}>= \pm k(\sinh t \sin \varphi, \sinh t \cos \varphi, \cosh t)
\end{aligned}
$$

The probability distribution of the area in a state of given $(m, \lambda)$ will be given by the modulus square of the overlaps of $\psi_{\frac{n}{2}}^{(n \rho) \frac{n}{2}}$ or $\psi_{ \pm \frac{n}{2}}^{(n \rho) k \pm}$ with $\psi_{ \pm \frac{n}{2} \lambda}^{(n \rho)}$. These have been calculated [22] [32] [31], see Appendix B, and have indeed a maximum at $\lambda \approx \frac{\rho}{2}$, but further studies are needed to judge whether these staes provide a viable alternative.

\section{$4 \quad$ A detailed example of triangulation.}

A triangulation evolving a pentachoron at $\mathrm{t}=0$ (abcde) to a later pentachoron at $\mathrm{t}=1$ $\left(a^{\prime} b^{\prime} c^{\prime} d^{\prime} e^{\prime}\right)$ can be realized connecting with edges all the vertices of the first to the vertices of the second, but omitting the edges $\left(a a^{\prime}\right),\left(b b^{\prime}\right),\left(c c^{\prime}\right),\left(d d^{\prime}\right),\left(e e^{\prime}\right)$; in this way we realize a division of the spacetime $S^{3} \otimes R$ between $\mathrm{t}=0$ and $\mathrm{t}=1$ in 304 -simplices. This triangulation can be proved to be orientable, i.e. it can be organized so that each of the 70 tetrahedron is in two 4-simplices with opposite orientation, for ex.

$$
\begin{array}{cccccc}
\left(a b c d e^{\prime}\right): & a b c d & a b e^{\prime} c & a b d e^{\prime} & a c e^{\prime} d & b c d e^{\prime} \\
\left(a b d c^{\prime} e^{\prime}\right): & a b d c^{\prime} & a b e^{\prime} d & a b c^{\prime} e^{\prime} & a d e^{\prime} c^{\prime} & b d c^{\prime} e^{\prime}
\end{array}
$$


The face dual to the triangle $\left(a b e^{\prime}\right)$ will be made of the following four steps:

$$
\begin{aligned}
& \left(a b c d e^{\prime}\right) \quad\left(a b d c^{\prime} e^{\prime}\right) \quad\left(a b e^{\prime} c^{\prime} d^{\prime}\right) \quad\left(a b c e^{\prime} d^{\prime}\right) \quad\left(a b c d e^{\prime}\right) \\
& a b e^{\prime} c, a b d e^{\prime} \rightarrow a b e^{\prime} d, a b c^{\prime} e^{\prime} \rightarrow a b e^{\prime} c^{\prime}, a b d^{\prime} e^{\prime} \rightarrow a b e^{\prime} d^{\prime}, a b c e^{\prime} \rightarrow a b e^{\prime} c, a b d e^{\prime}
\end{aligned}
$$

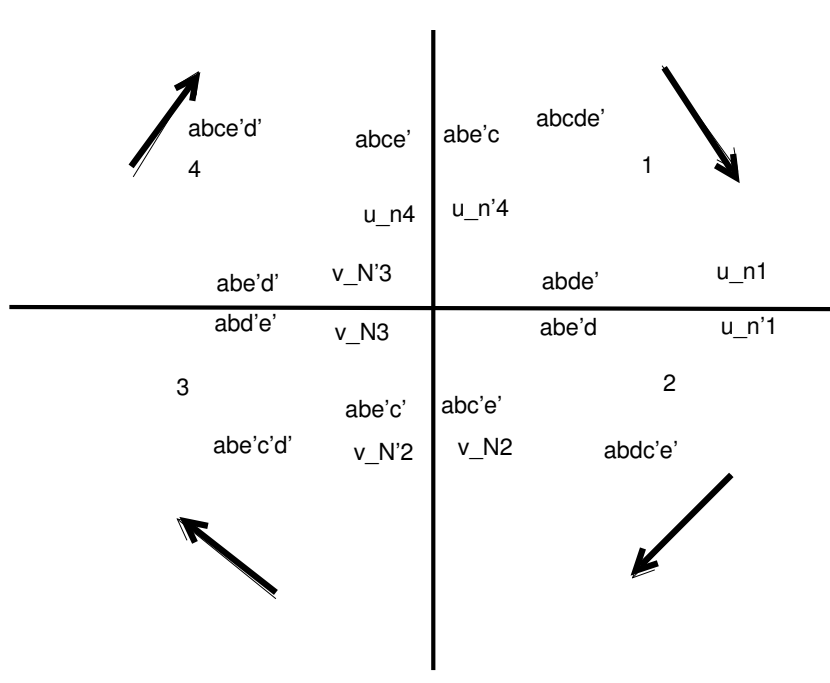

One can see that for ex. tetrahedron $\left(a b d e^{\prime}\right)=$ $\left(a b c d e^{\prime}\right) \cap\left(a b d c^{\prime} e^{\prime}\right)$, and that within $\left(a b c d e^{\prime}\right)$ triangle $t=\left(a b e^{\prime}\right)=\left(a b e^{\prime} c\right) \cap\left(a b d e^{\prime}\right)$. 4-simplices $\left(a b c d e^{\prime}\right),\left(a b d c^{\prime} e^{\prime}\right)$ have different frames, and triangle $t=\left(a b e^{\prime}\right)$ different area tensor, say $S_{t}^{I J}, S_{t}^{\prime I J}$ but $S_{t}^{I J} S_{t I J}=$ $S_{t}^{\prime I J} S_{t I J}^{\prime}$. The two frames are connected by a Lorentz transformation; the product of the four successive transformations, or $\mathrm{SL}(2, \mathrm{C})$ elements, gives the contribution of the face. Each transformation can be split for ex. $g_{\left(a b c d e^{\prime}\right) \rightarrow\left(a b d c^{\prime} e^{\prime}\right)}=g_{\left(a b d e^{\prime}\right)} \epsilon g_{a b e^{\prime} d}$ (the $\epsilon$ accounts for the reversal of orientation), so that the various contributions can be grouped in a product of 'vertex functions', one per 4simplex.

Can we assume that all the tetrahedra involved are space-like? To get an idea, consider the various 4-simplices involved in our model triangulation, and to simplify the analysis as much as possible assume that all edges at time zero have (length) ${ }^{2} l^{2}$, edges at time 1 (length) $)^{2} l^{\prime 2}$, edges between time 0 and time 1 (length) ${ }^{2}=d^{2}$. The centre of a bottom tetrahedron, say (abcd), is therefore at distance $\sqrt{\frac{3}{8}} l$ from any of its vertices, and for the two slices to be separated it must be $d^{2}<\frac{3}{8} l^{2}$; by the same argument applied to a top tetrahedron $d^{2}<\frac{3}{8} l^{\prime 2}$. To go further we can use Brewin's algorithm[34], or Görlich's explicit parametrization 33 . I find the following for the normals to the tetrahedra and the $(\text { area })^{2}$ of the triangles :

$(4,1):\left(a b c d e^{\prime}\right)$

$$
\begin{aligned}
V_{(a b c d)}^{2} & =-\frac{1}{2} l^{6} ; V_{\left(a b e^{\prime} c\right)}^{2}=\ldots=V_{\left(b c d e^{\prime}\right)}^{2}=\frac{3}{4} l^{4}\left(\frac{1}{3} l^{2}-d^{2}\right) ; \\
S_{d b c}^{2} & =S_{c a d}^{2}=S_{b a c}^{2}=S_{a b d}^{2}=\frac{3}{8} l^{4}, \quad S_{d c e}^{2}=\ldots=S_{e a c}^{2}=\frac{l^{2}}{2}\left(d^{2}-\frac{1}{4} l^{2}\right) .
\end{aligned}
$$

(here $S^{2}:=S^{I J} S_{I J}$ ). As we have seen, if $d^{2}=\frac{3}{8} l^{2}$ the two hypersurfaces are stuck together; they come apart as $d^{2}$ decreases, and all the tetrahedra are space-like, but four of them become light-like when $d^{2}=\frac{1}{3} l^{2}$, and time-like for still smaller $d^{2}$; six (area $)^{2}$ vanish at $d^{2}=\frac{1}{4} l^{2}$ and become negative for smaller $d^{2}$. So in the 'good' interval $\frac{3}{8} l^{2}>d^{2}>\frac{1}{4} l^{2}$ all $S^{2}$ are positive, and for $\frac{3}{8} l^{2}>d^{2}>\frac{1}{3} l^{2}$ all tetrahedra are space-like. Of course the same analysis applies to the $(1,4)$ four simplices, with $l \leftrightarrow l^{\prime}$. 
$(3,2):\left(a b d c^{\prime} e^{\prime}\right)$

$$
\begin{aligned}
V_{\left(a b d c^{\prime}\right)}^{2} & =V_{\left(a b e^{\prime} d\right)}^{2}=\frac{3}{4} l^{4}\left(\frac{1}{3} l^{2}-d^{2}\right) ; \quad V_{\left(a b c^{\prime} e^{\prime}\right)}^{2}=V_{\left(a d e^{\prime} c^{\prime}\right)}^{2}=V_{\left(b d c^{\prime} e^{\prime}\right)}^{2}=l^{2} l^{\prime 2}\left(\frac{1}{4} l^{2}+\frac{1}{4} l^{\prime 2}-d^{2}\right) \\
S_{a b d}^{2} & =\frac{3}{8} l^{2} ; \quad S_{a c^{\prime} e^{\prime}}^{2}=S_{d c^{\prime} e^{\prime}}^{2}=S_{c^{\prime} b e^{\prime}}^{2}=\frac{l^{2}}{2}\left(d^{2}-\frac{1}{4} l^{\prime 2}\right) ; \quad S_{a b e^{\prime}}^{2}=\ldots=S_{d a e^{\prime}}^{2}=\frac{l^{2}}{2}\left(d^{2}-\frac{1}{4} l^{2}\right)
\end{aligned}
$$

We have to look at this case together with the previous ones and the $(2,3)$ case; given that $d^{2}$ is such that $d^{2}<\frac{3}{8} l^{2}, d^{2}<\frac{3}{8} l^{2}$, it must be $d^{2}<\frac{3}{16}\left(l^{2}+l^{\prime 2}\right)$; but then tetrahedra (abc'e'), (bdc'e'), (ade'c') cannot be space-like, because that would require $d^{2}>\frac{1}{4}\left(l^{2}+l^{\prime 2}\right)$. We cannot therefore have this triangulation made exclusively of space-like tetrahedra. On the other hand the (area) ${ }^{2}$ of the triangles stay positive provided $d^{2}>\frac{1}{4} l^{2}, d^{2}>\frac{1}{4} l^{2}$. Notice the role that the various tetrahedra play in the face dual to (abe') in (4.29).

It may be that our simplifying assumptions are too strong, and that one can find a choice of lenghts which makes all tetrahedra space-like. I find it encouraging that one can at least assume that all (area) ${ }^{2}$ are positive, which is what matters. It may also be that larger triangulations do not have such tight constraint; this would require some experimenting that I have not attempted.

For the face dual to the triangle $\left(a b e^{\prime}\right)$ one would therefore find, on the assumption that tetrahedra $\left(a b c e^{\prime}\right),\left(a b d e^{\prime}\right)$ are space-like, tetrahedra $\left(a b c^{\prime} e^{\prime}\right),\left(a b d^{\prime} e^{\prime}\right)$ time-like, simplifying as much as possible the notation:

$$
\begin{gathered}
f_{\left(a b e^{\prime}\right)}=c \cdot \psi_{j, \gamma j}^{\dagger} u_{\mathbf{n}_{4}^{\prime}}^{-1} g_{4}^{\prime-1} \epsilon g_{1} u_{\mathbf{n}_{1}} \psi_{j, \gamma j} \psi_{j, \gamma j}^{\dagger} u_{\mathbf{n}_{1}^{\prime}}^{-1} g_{1}^{\prime-1} \epsilon g_{2} v_{N_{2}} \psi_{j, \gamma j} \psi_{j, \gamma j}^{\dagger} v_{N_{2}^{\prime}}^{-1} g_{2}^{\prime-1} \epsilon g_{3} v_{N_{3}} \psi_{j, \gamma j} \\
\cdot \psi_{j, \gamma j}^{\dagger} v_{N_{3}^{\prime}}^{-1} g_{3}^{\prime-1} \epsilon g_{4} u_{\mathbf{n}_{4}} \psi_{j, \gamma j}
\end{gathered}
$$

Here I have written $\psi_{j, \gamma j}$ for $\psi_{\frac{n}{2}, \gamma \frac{n}{2}}^{\left(n_{f} \rho_{f}\right)}$, and the representative of the $\operatorname{SL}(2, \mathbb{C})$ elements $\mathcal{D}^{\left(n_{f} \rho_{f}\right)}(g), \ldots$ simply as $g, \ldots$.

The 'vertex function' of a 4-simplex, say $\left(a b c d e^{\prime}\right)$ is the product of the factors corresponding to its five tetrahedra, integrated over the group variables, i.e. the product of matrix elements between data for the 10 triangles. Because of the overall $\mathrm{SL}(2, \mathbb{C})$ invariance, one of the group integration can be suppressed, and the final expression is finite. To calculate the partition function for the triangulation the product of the vertex functions should finally be summed over the $n_{f}$.

\section{Conclusions}

Spinors are a valuable tool to formulate the spin-foam theory, and they might allow the construction of calculable models. They give a considerable simplification of the various technical problems, which have so far hidden the basic simplicity of the model; hopefully, they might be instrumental in going beyond the simple picture of space-time at the basis of it. The use of the spinor basis for the quantum theory needs, and perhaps deserves further study.

\section{Acnowledgment}

I am very grateful to Dimitri Marinelli for the many long discussions we have had over the years, among other things on the subject of this work, and for help with some calculations, and to Eugenio Bianchi for telling me about [22] and sending me a copy of it. 


\section{Appendix A: 4-vectors, tensors, spinors.}

I collect here the various definitions and identities used in the text.

Dealing with spinors, I use repeatedly the following identities for the Pauli matrices $\sigma_{a}$ and the matrix $\epsilon=i \sigma_{2}$ :

$\epsilon \sigma_{a} \epsilon^{-1}=-\sigma_{a}^{T} ; \quad \sigma_{a \alpha \beta} \sigma_{a \gamma \delta}=\delta_{\alpha \beta} \delta_{\gamma \delta}-2 \epsilon_{\alpha \gamma} \epsilon_{\beta \delta} ; \quad t_{\alpha} \epsilon_{\alpha \beta} u_{\beta} v_{\gamma}+u_{\alpha} \epsilon_{\alpha \beta} v_{\beta} t_{\gamma}+v_{\alpha} \epsilon_{\alpha \beta} t_{\beta} u_{\gamma}=0$.

For the 4-d sigma matrices we have:

$$
\begin{aligned}
\sigma^{I} & =\left(1, \sigma_{a}\right), \quad \widetilde{\sigma}^{I}=\left(-1, \sigma_{a}\right)=-\epsilon \bar{\sigma}^{I} \epsilon^{-1} ; \\
\eta^{I J} & =\frac{1}{2} \operatorname{Tr}\left(\sigma^{I} \widetilde{\sigma}^{J}\right) ; \quad \sigma_{I} \widetilde{\sigma}_{J}+\sigma_{J} \widetilde{\sigma}_{I}=2 \eta_{I J} ; \quad \frac{1}{2} \epsilon^{I J K L} \sigma_{K} \widetilde{\sigma}_{L}=i \sigma^{[I} \widetilde{\sigma}^{J]} .
\end{aligned}
$$

The last equation can be used to project the selfdual/antiselfdual or the left/right components of an antisymmetric tensor:

$$
\sigma_{I} \widetilde{\sigma}_{J} J^{I J}=\sigma_{I} \widetilde{\sigma}_{J} J_{+}^{I J}=4 i \sigma_{a} J_{a}^{L}, \quad \tilde{\sigma}_{I} \sigma_{J} J^{I J}=\tilde{\sigma}_{I} \sigma_{J} J_{-}^{I J}=4 i \sigma_{a} J_{a}^{R}
$$

where $J_{ \pm}^{I J}=\frac{1}{2}\left(J^{I J} \mp i^{*} J^{I J}\right)$. I have used the identities, valid for an arbitrary 4-vector $V^{I}$ :

$$
V_{M} \widetilde{\sigma}_{I} J_{+}^{M I}=i \widetilde{\sigma}_{I} V^{I} \sigma_{a} J_{a}^{L} ; \quad V_{M} \widetilde{\sigma}_{I} J_{-}^{M I}=-i \sigma_{a} J_{a}^{R} \widetilde{\sigma}_{I} V^{I}
$$

Proof: from the definitions we have $J_{+}^{a b}=\epsilon_{a b c} J_{c}^{L}, J_{+}^{0 a}=-i J_{a}^{L}$; then:

$$
\begin{aligned}
V_{M} \widetilde{\sigma}_{I} J_{+}^{M I} & =V_{a} J_{+}^{a 0}+V_{0} \sigma_{a} J_{+}^{0 a}+V_{b} \sigma_{c} J_{+}^{b c}=i V_{a} J_{a}^{L}-i V_{0} \sigma_{a} J_{a}^{L}-V_{b} \sigma_{c} \epsilon_{c b a} J_{a}^{L}= \\
& =i V_{a} J_{a}^{L}-i V_{0} \sigma_{a} J_{a}^{L}+i V_{b}\left(\sigma_{b} \sigma_{a}-\delta_{a b}\right) J_{a}^{L}=i\left(V^{0}+\sigma_{b} V^{b}\right) \sigma_{a} J_{a}^{L} .
\end{aligned}
$$

The correspondence between spinor and vector representations of $\operatorname{SL}(2, \mathbb{C})$ is fixed by:

$$
g \sigma_{I} g^{\dagger}=\sigma_{J} \Lambda_{I}^{J} ; \quad g^{\dagger-1} \widetilde{\sigma}_{I} g^{-1}=\widetilde{\sigma}_{J} \Lambda_{I}^{J} ; \quad g \sigma_{a} g^{-1}=\sigma_{b} O_{b a},
$$

where the orthogonal matrix $O_{a^{\prime} a}$ is the representative of $g$ in the $(1,0)$ representation. The action of $\mathrm{SL}(2, \mathbb{C})$ on 4 -vectors and on the basic spinors is given by:

$$
T_{g} \cdot u=u g ; \quad T_{g} \cdot t=t g^{\dagger-1} ; \quad T_{g} \cdot X^{I}=X^{I^{\prime}} \Lambda_{I^{\prime}}^{I} ; \quad T_{g} \cdot\left(u \sigma_{I} \bar{u}\right)=\left(u \sigma_{I^{\prime}} \bar{u}\right) \Lambda_{I^{\prime}}^{I}
$$

These transformations are implemented through the $\mathrm{SL}(2, \mathbb{C})$ invariant Poisson brackets $\left\{t_{\alpha}, \bar{u}_{\beta}\right\}=-i \delta_{\alpha \beta} ; \quad\left\{u_{\beta}, \bar{t}_{\alpha}\right\}=-i \delta_{\alpha \beta}$, or

$$
\{f, h\}=i \frac{\partial f}{\partial \bar{t}_{\alpha}} \frac{\partial h}{\partial u_{\alpha}}-i \frac{\partial f}{\partial u_{\alpha}} \frac{\partial h}{\partial \bar{t}_{\alpha}}-i \frac{\partial f}{\partial t_{\alpha}} \frac{\partial h}{\partial \bar{u}_{\alpha}}+i \frac{\partial f}{\partial \bar{u}_{\alpha}} \frac{\partial h}{\partial t_{\alpha}},
$$

and generators:

$$
J_{a}^{L}=-\frac{1}{2} u \sigma_{a} \bar{t}, \quad J_{a}^{R}=-\frac{1}{2} t \sigma_{a} \bar{u} ; \quad\left\{J_{a}^{L, R}, J_{b}^{L, R}\right\}=\epsilon_{a b c} J_{c}^{L, R}, \quad\left\{J_{a}^{L}, J_{b}^{R}\right\}=0 .
$$

One finds:

$$
\left\{u_{\alpha}, J_{a}^{L}\right\}=\frac{i}{2} u_{\beta} \sigma_{a \beta \alpha} ; \quad\left\{f(u, \bar{u}), J_{a}^{L}\right\}=\frac{i}{2} u_{\beta} \sigma_{a \beta \alpha} \frac{\partial f}{\partial u_{\alpha}} ; \quad\left\{f(u, \bar{u}), J_{a}^{R}\right\}=\frac{i}{2} \frac{\partial f}{\partial \bar{u}_{\alpha}} \sigma_{a \alpha \beta} \bar{u}_{\beta}
$$


and therefore, if $g=1+i \epsilon_{a} L_{a}+i \eta_{a} K_{a}=1+\left(i \epsilon_{a}+\eta_{a}\right) J_{a}^{L}+\left(i \epsilon_{a}-\eta_{a}\right) J_{a}^{R} \simeq 1+\frac{i}{2}\left(\epsilon_{a} \sigma_{a}+i \eta_{a} \sigma_{a}\right)$

$$
T_{(\epsilon \eta)} \cdot f(u, \bar{u})=\frac{i}{2}\left(i \epsilon_{a}+\eta_{a}\right) u_{\beta} \sigma_{a \beta \alpha} \frac{\partial f}{\partial u_{\alpha}}+\frac{i}{2}\left(i \epsilon_{a}-\eta_{a}\right) \frac{\partial f}{\partial \bar{u}_{\alpha}} \sigma_{a \alpha \beta}
$$

To go to finite group elements:

$$
e^{\left\{\cdot, v_{a} J_{a}^{L}+w_{a} J_{a}^{R}\right\}} f(u, \bar{u})=f\left(u e^{\frac{i}{2} v_{a} \sigma_{a}}, \bar{u} e^{\frac{i}{2} w_{a} \sigma_{a}}\right) .
$$

\section{Appendix B: The Overlap}

The overlap of $\psi_{\frac{n}{2}}^{(n \rho) \frac{n}{2}}, \psi_{ \pm \frac{n}{2}}^{(n \rho) k \pm}$ with $\psi_{ \pm \frac{n}{2} \lambda}^{(n \rho)}$ can be calculated considering the states of the three types considered, with $\frac{n}{2}=j=k, m= \pm n$; the expressions of $d_{\frac{n}{2} \frac{n}{2}}^{\frac{n}{2}}(\theta), b_{ \pm \frac{n}{2} \pm \frac{n}{2}}^{\frac{n}{2}}(t)$ are very simple. Listing them, with the parametrizations of $P^{I}$ :

$$
\begin{gathered}
\psi_{\frac{n}{2}}^{(n \rho) \frac{n}{2}}=C_{a} e^{\left(i \frac{\rho}{2}-1\right) a} d_{\frac{n}{2} \frac{n}{2}}^{\frac{n}{2}}(\theta)=C_{a} e^{\left(i \frac{\rho}{2}-1\right) a}\left(\frac{1+\cos \theta}{2}\right)^{\frac{n}{2}} ; \quad P^{I}=e^{a}(1, \sin \theta \cos \varphi, \sin \theta \sin \varphi, \cos \theta) \\
\psi_{ \pm \frac{n}{2}}^{(n \rho) k \pm}=C_{b} e^{\left(i \frac{\rho}{2}-1\right) b} b_{ \pm \frac{n}{2} \pm \frac{n}{2}}^{\frac{n}{2}}(t)=C_{b} e^{\left(i \frac{\rho}{2}-1\right) b}\left(\frac{2}{1+\cosh t}\right)^{\frac{n}{2}} ; P^{I}=e^{b}(\cosh t, \sinh t \cos \varphi, \sinh t \sin \varphi, 1) \\
\psi_{ \pm \frac{n}{2} \lambda}^{(n \rho)}=C_{c} e^{\left(i \frac{\rho}{2}-1\right) c} e^{i \lambda u} ; \quad P^{I}=e^{c}(\cosh u, \cos \varphi, \sin \varphi, \sinh u) \\
\frac{d^{3} P}{2 P(2 \pi)^{3}}=\frac{e^{2 a} d a \sin \theta d \theta d \varphi}{2(2 \pi)^{3}}=\frac{e^{2 b} \sinh t d t d b d \varphi}{2(2 \pi)^{3}}=\frac{e^{2 c} d c d u d \varphi}{2(2 \pi)^{3}} .
\end{gathered}
$$

However, as explained in the text, one omits the integration in $d a$ or $d b$ or $d c$. Equating the expressions of $P^{I}$ :

$$
e^{a}=e^{b} \cosh t=e^{c} \cosh u ; \quad \cos \theta=\frac{\sinh u}{\cosh u} ; \quad \cosh t=\frac{\cosh u}{\sinh u} ; \quad e^{b}=e^{c} \sinh u
$$

Then I find, in the two cases:

$$
\begin{aligned}
<\psi_{\frac{n}{2} \lambda}^{(n \rho)} \mid \psi_{\frac{n}{2}}^{(n \rho) \frac{n}{2}}> & =\int \frac{e^{2 c} d u 2 \pi}{2(2 \pi)^{3}} C_{c} C_{a} e^{\left(-i \frac{\rho^{\prime}}{2}-1\right) c} e^{-i \lambda u} e^{\left(i \frac{\rho}{2}-1\right) a}\left(\frac{1+\cos \theta}{2}\right)^{\frac{n}{2}}= \\
& =\frac{C_{c} C_{a}}{8 \pi^{2} 2^{n / 2}} \int d u \frac{e^{\left(-i \lambda+\frac{n}{2}\right) u}}{(\cosh u)^{1+\frac{n}{2}-i \frac{\rho}{2}}}= \\
& =\frac{C_{c} C_{a}}{8 \pi^{2} 2^{\left(i \frac{\rho}{2}\right)}} \frac{\Gamma\left(\frac{1}{2}+\frac{i}{2}\left(\lambda-\frac{\rho}{2}\right)\right) \Gamma\left(\frac{1}{2}+\frac{n}{2}-\frac{i}{2}\left(\lambda+\frac{\rho}{2}\right)\right)}{\Gamma\left(1+\frac{n}{2}-i \frac{\rho}{2}\right)} \\
<\psi_{ \pm \frac{n}{2} \lambda}^{(n \rho)} \mid \psi_{ \pm \frac{n}{2}}^{(n \rho) k \pm}> & =\int \frac{e^{2 c} d u 2 \pi}{2(2 \pi)^{3}} C_{c} C_{b} e^{\left(-i \frac{\rho^{\prime}}{2}-1\right) c} e^{-i \lambda u} e^{\left(i \frac{\rho}{2}-1\right) b}\left(\frac{2}{1+\cosh t}\right)^{\frac{n}{2}}= \\
& =\frac{C_{c} C_{b}}{8 \pi^{2}} 2^{n / 2} \int d u e^{-\left(i \lambda+\frac{n}{2}\right) u}(\sinh u)^{\frac{n}{2}+i \frac{\rho}{2}-1}= \\
& =\frac{C_{c} C_{b}}{8 \pi^{2} 2^{i \frac{\rho}{2}}} \frac{\Gamma\left(\frac{1}{2}+\frac{i}{2}\left(\lambda-\frac{\rho}{2}\right)\right) \Gamma\left(\frac{n}{2}+i \frac{\rho}{2}\right)}{\Gamma\left(\frac{1}{2}+\frac{n}{2}+\frac{i}{2}\left(\lambda+\frac{\rho}{2}\right)\right)}
\end{aligned}
$$


From these expressions we get that for $k \geq 1$, up to factors independent on $\lambda$ :

$$
\begin{aligned}
& \left|<\psi_{\frac{n}{2} \lambda}^{(n \rho)}\right| \psi_{\frac{n}{2}}^{(n \rho) \frac{n}{2}}>\left.\right|^{2} \sim \frac{1}{\cosh \pi \lambda+\cosh \pi \frac{\rho}{2}} \prod_{j=1}^{k} \frac{(2 j-1)^{2}+\left(\lambda+\frac{\rho}{2}\right)^{2}}{4}, \quad n=2 k ; \\
& \sim \frac{\left(\lambda-\frac{\rho}{2}\right)}{\sinh \pi \lambda-\sinh \pi \frac{\rho}{2}} \prod_{j=1}^{k}\left(j^{2}+\frac{\left(\lambda+\frac{\rho}{2}\right)^{2}}{4}\right), \quad n=2 k+1 . \\
& \left|<\psi_{ \pm \frac{n}{2} \lambda}^{(n \rho)}\right| \psi_{ \pm \frac{n}{2}}^{(n \rho) k \pm}>\left.\right|^{2} \sim \frac{\cosh \frac{\pi}{2}\left(\lambda+\frac{\rho}{2}\right)}{\cosh \frac{\pi}{2}\left(\lambda-\frac{\rho}{2}\right)} \prod_{j=1}^{k} \frac{4}{(2 j-1)^{2}+\left(\lambda+\frac{\rho}{2}\right)^{2}}, \quad n=2 k \\
& \sim \frac{\sinh \frac{\pi}{2}\left(\lambda+\frac{\rho}{2}\right)}{\frac{\pi}{2}\left(\lambda+\frac{\rho}{2}\right) \cosh \frac{\pi}{2}\left(\lambda-\frac{\rho}{2}\right)} \prod_{j=1}^{k} \frac{1}{j^{2}+\frac{\left(\lambda+\frac{\rho}{2}\right)^{2}}{4}}, \quad n=2 k+1 .
\end{aligned}
$$

(for $k=0$, i.e. for $n=0$ or $n=\frac{1}{2}$, same expressions with no products).

For $k=1, n=2$ I find with the first of these expressions $\langle\lambda\rangle=\frac{\rho}{4}$ and

$<\left(\lambda-\langle\lambda>)^{2}\right\rangle=\frac{3}{5}+\frac{3}{20}\left(\frac{\rho}{2}\right)^{2}$, and I presume that the other expressions would give similar results. I do not know of a good approximation to investigate the behaviour for large $n$.

\section{References}

[1] J. W. Barrett and L. Crane, "A Lorentzian signature model for quantum general relativity", Class. Quant. Grav. 17 (2000) 3101, arXiv:gr-qc/9904025.

[2] S. Holst, "Barbero's Hamiltonian derived from a generalized Hilbert-Palatini action", Phys. Rev. D 53 (1996) 5966, gr-qc/9511026

[3] M. Dupuis, L. Freidel, E. Livine, S. Speziale, 'Holomorphic Lorentzian Simplicity Constraints', arXiv:1107.5274.

[4] J. Engle, R. Pereira, C. Rovelli and E. Livine "LQG vertex with finite Immirzi parameter", Nucl. Phys. B799 (2008) 136-149, arXiv:0711.0146

[5] E. Livine, S. Speziale. "A new spin-foam vertex for quantum gravity", Phys. Rev. D 76 (2007) 084028, arXiv:0705.0674.

[6] L. Freidel, K. Krasnov, "A new spin-foam model for 4-d quantum gravity' Class.Quant.Grav. 25 (2008) 125018, arXiv:0708.1595.

[7] Eugenio Bianchi, Carlo Rovelli, Francesca Vidotto, 'Towards spin-foam cosmology', Phys.Rev. D82 084035 (2010), arXiv: 1003.3483.

Francesca Vidotto, 'Many-nodes/many-links spin-foam: the homogeneous and isotropic case', Class.Quant.Grav. 28 (20110) 245005, arXiv:1107.2633.

Muxin Han, 'Covariant loop quantum gravity, low energy perturbation theory and Einstein gravity', arXiv1308.4063.

[8] J. Ambjorn, J. Jurkiewicz, R. Loll, 'Quantum Gravity, or The Art of Building Spacetime', in "Approaches to Quantum Gravity", ed. D.Oriti, Cambridge University Press.

[9] F. Conrady, "Spin foams with timelike surfaces", Class. Quant. Grav. 27 (2010) 155014, arXiv:1003.5652. 
[10] F. Conrady, J. Hnybida, "A spin foam model for general Lorentzian 4-geometries", Class. Quant. Grav. 27 (2010) 185011, arXiv:1002.1959.

[11] J. W. Barrett, M. Galassi, W. A. Miller, R. D. Sorkin, P. A. Tuckey, R. M. Williams, "A Parallelizable Implicit Evolution Scheme for Regge Calculus", Int.J.Theor.Phys 36 (1997) 815, arXiv:gr-qc/9411008.

[12] S. Jordan and R. Loll, "De Sitter universe from Causal Dynamic Triangulation without preferred foliation", arXiv 1307.5469.

[13] Yasha Neiman, "Polyhedra in spacetime from null vectors", arXiv:1308.1982.

[14] I. M. Gel'fand, Naum Vilenkin, Generalized Functions. Volume 4: Applications of Harmonic Analysis, Academic Press, 1964;

M. A. Naimark, "Linear representations of the Lorentz group", Pergamon Press, 1964.

[15] John W. Barrett, Richard J. Dowdall, Winston J. Fairbairn, Frank Hellmann, Roberto Pereira, "Lorentzian spin foam amplitudes: graphical calculus and asymptotics", Class. Quant. Grav. 27, 165009 (2010) arXiv:0907.2440.

[16] D. Oriti, "The Feynman propagator for quantum gravity: spin foams, proper time, orientation, causality and timeless-ordering" Braz.J.Phys. 35 (2005) 481, gr-qc/0412035.

'The Feynman propagator for spin foam quantum gravity', Phys.Rev.Lett. 94 (2005) 111301, gr-qc/0410134;

E. R. Livine and D. Oriti , "Implementing causality in the spin foam quantum geometry", Nucl.Phys. B663 (2003) 231, gr-qc/0210064.

[17] Carlo Rovelli, Edward Wilson-Ewing, "Discrete Symmetries in Covariant LQG", arXiv: 1205.0733

Marios Christodoulou, Aldo Riello, Carlo Rovelli "How to detect an anti-spacetime', arXiv:1206.3903.

Marios Christodoulou, Miklos Langvik, Aldo Riello, Christian Röken, Carlo Rovelli, "Divergences and Orientation in Spinfoams", arXiv:1207.5156.

[18] Jonathan Engle, 'A proposed proper EPRL vertex amplitude', Phys. Rev. D 87 084048 (2013), arXiv:1111.2865; 'A spin-foam vertex amplitute with the correct semiclassical behaviour', Phys. Lett. B724 (2013) 333, arXiv:1201.2187

[19] Marko Vojinović, "Cosine problem in EPRL/FK spinfoam model", arXiv:1307.5352.

[20] Simone Speziale, Mingyi Zhang, 'Null twisted geometries', arXiv:1311.3279

[21] Ya. A. Smorodinskii and M. Huszár, "Representations of the Lorentz group and generalization of the helicity states", Theoret. Math. Phys. 4867 (1970).

[22] M. Huszár "Angular momentum and unitary spinor bases of the Lorentz group", Acta Physica Academica Scientiarum Hungaricae 30 (1971) 241.

[23] Moshe Carmeli, "Group Theory and General Relativity", (McGraw Hill, 1977).

[24] V. Bargmann, "Irreducible unitary representatons of the Lorentz group", Annals of Math., 48, 568 (1947).

[25] W. Ruhl, "The Lorentz Group and Harmonic Analysis", (W.A. Benjamin, New York, 1970). 
[26] F. Conrady, J. Hnybida, " Unitary irreducible representations of $\mathrm{SL}(2, \mathbb{C})$ in discrete and continuous SU(1,1) bases", J. Math. Phys. 52 (2011) 012501, arXiv:1007.0937

[27] A. Sciarrino, M. Toller, "Decomposition of unitary representatons of the group SL(2,C) restricted to the subgroup SU(1,1)", J. Math. Phys. 8, 1252 (1967) .

[28] N. Mukunda, "Unitary representations of the homogeneous Lorentz group in an O(2,1) basis", J.Math. Phys. 9 (1968) 50.

[29] J. S. Lomont, H. E. Moses, "Simple realization of the infinitesimal generators of the proper ortochronous inhomogeneous Lorentz group for mass zero", J.Math. Phys. 3 (1962) 405.

[30] E.G. Kalnins, "Unitary representations of the homogeneous Lorentz group in a $\mathrm{O}(1,1) \mathrm{O}(2)$ basis and some applications to relativistic equations", J.Math. Phys. 13, 1304 (1972).

[31] S.J. Chang, L. O'Raifertaigh, "Unitary representation of $\mathrm{SL}(2, \mathbb{C})$ in an $\mathrm{E}(2)$ basis", J. Math. Phys. 10, 21 (1969)

[32] Eugenio Bianchi, "Entropy of Non-Extremal Black Holes from Loop Gravity", arXiv:1204.5122

[33] A. Görlich, PhD thesis, Jagiellonian University, September 2010, arXiv:1111.6938.

[34] L. Brewin. "Fast algorithms for computing defects and their derivatives in the Regge calculus", Class. Quant. Grav. arXiv:1011.1885. 